quatrième série-tome 42 fascicule 2 mars-avril 2009

$$
\begin{aligned}
& \text { ANNALES } \\
& \text { SCIENTIFIQUES } \\
& \text { de } \\
& \text { L'ECOLE } \\
& \text { NORMALE } \\
& \text { SUPÉRIEURE }
\end{aligned}
$$

Fabrice PLANCHON \& Luis VEGA

Bilinear virial identities and applications 
Ann. Scient. Éc. Norm. Sup.

$4^{\text {e }}$ série, t. 42,2009 , p. 261 à 290

\title{
BILINEAR VIRIAL IDENTITIES AND APPLICATIONS
}

\author{
By FAbrice PLANCHON* and Luis VEGA**
}

\begin{abstract}
We prove bilinear virial identities for the nonlinear Schrödinger equation, which are extensions of the Morawetz interaction inequalities. We recover and extend known bilinear improvements to Strichartz inequalities and provide applications to various nonlinear problems, most notably on domains with boundaries.

RÉSUMÉ. - On démontre des identités de type viriel bilinéaire pour l'équation de Schrödinger nonlinéaire, qui peuvent être vues comme des extensions des inégalités d'interaction de Morawetz. Ceci permet de retrouver et d'étendre des raffinements bilinéaires des inégalités de Strichartz, et nous donnons également des applications à plusieurs problèmes non-linéaires, notamment sur les domaines à bord.
\end{abstract}

\section{Introduction}

Dispersive estimates are known to be an essential tool in dealing with low regularity wellposedness issues for the nonlinear Schrödinger equation. Among the most useful ones are Strichartz inequalities: starting with [26], they were completed by [15] and finally by [19]. As space-time bounds for solutions to the linear Schrödinger equation in $\mathbb{R}^{n}$, they are closely related to the Fourier restriction problem in harmonic analysis, and as such heavily rely on the use of Fourier transform techniques. Extensions of these inequalities to more complicated geometrical settings have been the subject of intense research over the last decade, to the point where quoting all possible references would fill this page. It should be noted that these works are based on appropriate refinements of the $\mathbb{R}^{n}$ case, through Fourier Integral Operator, FBI, wave packet or any appropriate microlocal generalizations of Fourier analysis (for a notable exception using vector field methods, see [23]). On the other hand, one has virial type identities, of which the Morawetz identity (proved by Lin-Strauss [21]) is perhaps the most well-known: such identities have two key features, they are obtained by integration

\footnotetext{
* Partially supported by A.N.R. grant ONDE NON LIN.

** Partially supported by grant MTM 2007-62186.
} 
by parts and they usually apply to the nonlinear equation. We remark that the local smoothing effect, which came much later and was first observed in the flat case (see [14], [24], [29]), may be seen as part of this category as well, though proofs usually require a sophisticated "integration by parts" involving pseudo-differential operators or resolvent methods. A new kind of inequality was introduced in [12], the Morawetz interaction inequality, which seemed to have the benefit of both worlds: one may recover a specific, non-sharp Strichartz estimate and it also applies to the nonlinear equation (providing an essential tool to solve the $H^{1}$-critical defocusing NLS in 3D, [13]). Subsequent developments include a curved space version ([17]) and a quartic interaction inequality for NLS on $\mathbb{R}$ ([11]).

In the present work, we explore a different direction, which builds upon the understanding of the local smoothing effect and its fundamentally 1D nature. This naturally leads to a new set of identities with several interesting consequences:

- in 1D, one recovers, by a simple argument, an identity of [22], which implies the Fefferman-Stein inequality in its bilinear version; from there the (almost) full set of Strichartz/maximal function estimates may be derived. More importantly, we get a nonlinear identity.

- In 2D and higher, one obtains an $L_{t, x}^{2}$-based estimate for the charge density. (This would correspond, w.r.t. scaling, to a sharp Strichartz estimate in 2D.) More interestingly, one may derive from our result Bourgain's bilinear improvement ([3]).

- All our identities apply to nonlinear equations, and have bilinear versions.

- Nothing but integration by parts is used in the proof: as such, these estimates extend to domains, provided one may control the boundary terms; in the case of Dirichlet boundary conditions, such control is provided by local smoothing.

- As an application to exterior domains, we improve the well-posedness theory to $H^{1}$-subcritical (subquintic) nonlinearities for $n=3$.

- Applications to scattering problems are straightforward, and this extends to 3D exterior domains, where no results were available to our knowledge and where we obtain scattering in the energy class for the defocusing cubic equation.

While presenting this work at Oberwolfach, we learned that similar results (namely a priori bound (2.9)) have been obtained simultaneously and independently by J. Colliander, M. Grillakis and N. Tzirakis, see [9] and [10]), through a different derivation.

Acknowledgments. - We thank N. Burq for various enlightenments about the Schrödinger equation on exterior domains, C. Zuily for pointing out an incomplete proof in an earlier version, as well as the referee for helpful comments and suggestions which greatly improved the presentation.

\section{Main results}

\subsection{The Schrödinger equation in $\mathbb{R}^{n}$}

Let $n \geq 1, p \in \mathbb{R}, p \geq 1, \varepsilon \in\{-1,0,1\}$, and $u$ is a solution to

$$
i \partial_{t} u+\Delta u=\varepsilon|u|^{p-1} u, \text { with } u_{\mid t=0}=u_{0} .
$$

$4^{\text {e }}$ SÉRIE - TOME $42-2009-\mathrm{N}^{\mathrm{o}} 2$ 
We will also need $v$, solution to

$$
i \partial_{t} v+\Delta v=\varepsilon|v|^{p-1} v, \text { with } v_{\mid t=0}=v_{0} .
$$

Let us define several quantities which will play a key role: for $n>1$ and given a function $f$, its Radon transform is

$$
R(f)(s, \omega)=\int_{x \cdot \omega=s} f d \mu_{s, \omega},
$$

where $\mu_{s, \omega}$ is the induced measure on the hyperplane $x \cdot \omega=s$. We set

$$
I_{\omega}(\varepsilon, u, v)=\int_{x \cdot \omega>y \cdot \omega}(x \cdot \omega-y \cdot \omega)|u|^{2}(x)|v|^{2}(y) d x d y .
$$

Remark that a simple computation leads to

$$
\partial_{t} I_{\omega}=i\left(\int_{x \cdot \omega>y \cdot \omega} \omega \cdot\left[\left(u \nabla_{x} \bar{u}-\bar{u} \nabla_{x} u\right)(x)|v(y)|^{2}-\left(v \nabla_{y} \bar{v}-\bar{v} \nabla_{y} v\right)(y)|u(x)|^{2}\right] d y d x\right) .
$$

We may now state our first result.

Theorem 2.1. - Let $\omega \in \mathbb{R}^{n}, n>1$, with $|\omega|=1$, u solution to (2.1). Then, with $x=x^{\perp}+s \omega$

$$
\begin{aligned}
& \text { (2.6) } \int_{s}\left|\partial_{s}\left(R\left(|u|^{2}\right)\right)(s, \omega)\right|^{2} d s+\varepsilon \frac{p-1}{p+1} \int_{s} R\left(|u|^{2}\right) R\left(|u|^{p+1}\right) d s \\
& +\int_{s} \int_{x^{\perp} \cdot \omega=0} \int_{y^{\perp} \cdot \omega=0}\left|u\left(x^{\perp}+s \omega\right) \partial_{s} u\left(y^{\perp}+s \omega\right)-u\left(y^{\perp}+s \omega\right) \partial_{s} u\left(x^{\perp}+s \omega\right)\right|^{2} d x^{\perp} d y^{\perp} d s \\
& =\frac{1}{4} \partial_{t}^{2} I_{\omega}(\varepsilon, u, u) .
\end{aligned}
$$

In other words, $I_{\omega}(\varepsilon, u, u)$ is a convex function in time.

In the specific $1 \mathrm{D}$ case, one has actually the following identity.

THeOREM 2.2. - Let $n=1, u, v$ two solutions to (2.1), (2.2), then

$$
4 \int_{x}\left|\partial_{x}(u \bar{v})\right|^{2} d x+2 \varepsilon \frac{p-1}{p+1} \int_{x}|u|^{2}|v|^{p+1}+|v|^{2}|u|^{p+1} d x=\partial_{t}^{2} I(\epsilon, u, v) .
$$

ReMARK 2.1. - Up to a doubling factor, $I_{\omega}$ may be recast as a Morawetz interaction functional (as introduced in [12]),

$$
\int \rho(x-y)|u|^{2}(x)|v|^{2}(y) d x d y
$$

with $\rho(x-y)=|x \cdot \omega-y \cdot \omega|$. Hence we have replaced the physical distance $|x-y|$ (which was the default choice in [12] and subsequent works) by its projection over a specified direction $\omega$. We chose our definition of $I_{\omega}$ as to emphasize trace terms which will later appear in the proof. In fact, we were led to $I_{\omega}$ by considering variations on the local smoothing, and we will come back to this point in Section 4.2.

In order to turn these bounds into useful nonlinear control, we use 
Proposition 2.2. - Let $\omega$ be fixed, then

$$
\left|\partial_{t} I_{\omega}\right| \leq\|u\|_{L_{x}^{2}}^{2}\|v\|_{\dot{H}^{\frac{1}{2}}}^{2}+\|v\|_{L_{x}^{2}}^{2}\|u\|_{\dot{H}^{\frac{1}{2}}}^{2} .
$$

As a consequence, when $\varepsilon=1$ (defocusing equation), we have an priori bound,

$$
\left.\left.\int_{\mathbb{R}} \int_{\mathbb{R}^{n}}\left\|\left.\left.\nabla\right|^{\frac{3-n}{2}}\left(|u|^{2}\right)\right|^{2} d x d t+\int_{\mathbb{R}} \int_{\mathbb{R}^{n}}\right\| \nabla\right|^{\frac{1-n}{2}}\left(|u|^{\frac{p+3}{2}}\right)\right|^{2} d x d t \lesssim \sup _{t \in \mathbb{R}}\|u\|_{L_{x}^{2}}^{2}\|u\|_{\dot{H}^{\frac{1}{2}}}^{2} .
$$

REMARK 2.3. - The right-hand side of (2.8) is very clearly not invariant by galilean transforms. The left-hand side, however, is.

REMARK 2.4. - The a priori estimate (2.9) was obtained simultaneously and independently by J. Colliander, M. Grillakis and N. Tzirakis [9, 10], through a direct derivation with the weight $\rho(x)=|x|$ but with a new commutator argument involving $\left[x,{\sqrt{-\Delta^{-(n-1)}}}^{-}\right.$and the local conservation laws for mass and momentum densities, overcoming the restriction to dimensions $n \geq 3$ from [12].

We now state a more general result: let

$$
I_{\rho}(u, v)=\int \rho(x-y)|u|^{2}(x)|v|^{2}(y) d x d y .
$$

Then

Theorem 2.3. - Let $\rho$ be a weight function such that its Hessian $H_{\rho}$ is positive; let

$F(u, v)(x, y)=\bar{v}(y) \nabla_{x} u(x)+u(x) \nabla_{y} \bar{v}(y)$ and $G(u, v)(x, y)=v(y) \nabla_{x} u(x)-u(x) \nabla_{y} v(y)$.

We have

$$
\begin{aligned}
\partial_{t}^{2} I_{\rho}= & 4 \int H_{\rho}(x-y)(F(u, v)(x, y), \bar{F}(u, v)(x, y)) d x d y \\
& +\varepsilon \frac{p-1}{p+1} \int|v|^{2}(y)\left(\Delta_{x} \rho\right)(x-y)|u|^{p+1}(x) d x d y \\
& +\varepsilon \frac{p-1}{p+1} \int|u|^{2}(x)\left(\Delta_{x} \rho\right)(x-y)|v|^{p+1}(y) d x d y
\end{aligned}
$$

Moreover, we may rewrite

$$
\int H_{\rho}(x-y)(F(u, v)(x, y), \bar{F}(u, v)(x, y)) d x d y=
$$

$\left.\int H_{\rho}(x-y)(G(u, v)(x, y), \bar{G}(u, v)(x, y)) d x d y+\int \Delta \rho(x-y) \nabla_{x}\left(|u|^{2}(x)\right) \cdot \nabla_{y}\left(|v|^{2}(y)\right)\right) d x d y$.

REMARK 2.5. - Notice that if we make $u=v$ in (2.13) and assume that the Fourier transform of $\Delta \rho$ is positive, we can bound each of the two terms in the right-hand side in terms of the left-hand side.

The above remark used in the particular case $\rho(z)=|z \cdot \omega|$ gives us the following corollary for the linear equation.

$4^{\mathrm{e}}$ SÉRIE - TOME $42-2009-\mathrm{N}^{\mathrm{o}} 2$ 
Theorem 2.4. - Given $\omega$ a unitary vector in $\mathbb{R}^{n}, n>1$ and $u, v$ any two solutions to (2.1) and (2.2) with $\epsilon=0$ (linear equation) we have

$$
\int_{t} \int_{s}\left|\partial_{s}(R(u \bar{v}))(s, \omega)\right|^{2} d s d t \lesssim I_{\omega}\left(\hat{u}_{0}, \hat{u}_{0}\right)+I_{\omega}\left(\hat{v}_{0}, \hat{v}_{0}\right)+I_{\omega}\left(\hat{u}_{0}, \hat{v}_{0}\right)
$$

with $I_{\omega}$ as given in (2.4).

REMARK 2.6. - We will see that this bilinear estimate implies Bourgain's bilinear refinement of Strichartz estimate from [3]. One may notice that (2.4) (and all identities involving the Radon transform) does not depend on the dimension $n$, in sharp contrast with (2.9), which gets worse with $n$ large.

\subsection{The Schrödinger equation on a domain $\Omega$}

Let $n \geq 1, p \in \mathbb{R}, p \geq 1, \varepsilon \in\{-1,0,1\}, \Omega \subset \mathbb{R}^{n}$ with a smooth boundary $\partial \Omega$, and $u$ is now the solution to

$$
i \partial_{t} u+\Delta u=\varepsilon|u|^{p-1} u, \text { with } u_{\mid \partial \Omega}=0
$$

Denote by

$$
M(u)=\int_{\Omega}|u|^{2} d x \text { and } E(u)=\frac{1}{2} \int_{\Omega}|\nabla u|^{2} d x+\frac{1}{p+1} \int_{\Omega}|u|^{p+1} d x
$$

the mass and energy which are conserved quantities: we will use $M$ and $E$ as shorter versions of $M(u)$ and $E(u)$. Notice that the Radon transform is still defined,

$$
R(f)(s, \omega)=\int_{x \cdot \omega=s \cap \Omega} f d \mu_{s, \omega} .
$$

We set

$$
I_{\rho}=\int_{x, y \in \Omega} \rho(x-y)|u|^{2}(x)|u|^{2}(y) d x d y .
$$

We may now state our result.

Theorem 2.5. - Let $\omega \in \mathbb{R}^{n}, n>1$, with $|\omega|=1$, and pick $\rho_{\omega}(z)=|z \cdot \omega|$, u solution to (2.15). Then, with $x=x^{\perp}+s \omega$

$$
\begin{aligned}
& \int_{s}\left|\partial_{s}\left(R\left(|u|^{2}\right)\right)(s, \omega)\right|^{2} d s+\varepsilon \frac{p-1}{p+1} \int_{s} R\left(|u|^{2}\right) R\left(|u|^{p+1}\right) d s \\
& \quad+\int_{s} \int_{x \cdot \omega=s} \int_{y \cdot \omega=s}\left|u\left(x^{\perp}+s \omega\right) \partial_{s} u\left(y^{\perp}+s \omega\right)-u\left(y^{\perp}+s \omega\right) \partial_{s} u\left(x^{\perp}+s \omega\right)\right|^{2} d x^{\perp} d y^{\perp} d s \\
& \quad-\int_{x \in \partial \Omega, y \in \Omega}|u|^{2}(y) \partial_{n} \rho_{\omega}(x-y)\left|\partial_{n} u\right|^{2}(x) d S_{x} d y=\partial_{t}^{2} I_{\rho_{\omega}} .
\end{aligned}
$$

We now illustrate how to obtain useful estimates from Theorem 2.5 when one has control of the boundary term. 
Proposition 2.7. - Let $\Omega$ be $\mathbb{R}^{n} \backslash \Sigma$, where $\Sigma$ is star-shaped and $\Sigma \Subset K, K$ compact. Assume moreover $\varepsilon=0,1$ (linear or defocusing) and $n \geq 3$. Then,

$$
\int_{0}^{T} \int_{x \in \partial \Omega}\left|\partial_{n} u\right|^{2} d S_{x} d t+\int_{0}^{T} \int_{K \backslash \Sigma}\left(|\nabla u|^{2}+|u|^{2}\right) d x d t \lesssim \sup _{t \in[0, T]}\|u\|_{\dot{H}_{0}^{\frac{1}{2}}(\Omega)}^{2} \lesssim(M E)^{\frac{1}{2}}
$$

REMARK 2.8. - In 2D, one may only obtain a local in time estimate for the defocusing equation, an issue related to the zero mode (or the failure of the Morawetz estimate in 2D) Hence, (2.20) will have an additional term $C(T)\left\|u_{0}\right\|_{L^{2}}^{2}$ on the right-hand side. We will not use such an estimate and therefore skip it.

As a consequence of Theorem 2.5 and Proposition 2.7, we have

Proposition 2.9. - Let $\Omega$ be $\mathbb{R}^{n} \backslash \Sigma$, where $\Sigma$ is star-shaped and $\Sigma \Subset K, K$ compact, and $n \geq 3$. Then, the solution $u$ to the defocusing ( $\varepsilon=1$ ) equation(2.15) verifies

$$
\left\|\left|\nabla_{x}\right|^{\frac{3-n}{2}}\left(|u|^{2}\right)\right\|_{L_{t, x}^{2}} \lesssim \sup _{t}\|u\|_{L^{2}(\Omega)}\|u\|_{\dot{H}_{0}^{\frac{1}{2}}} \lesssim M^{\frac{3}{4}} E^{\frac{1}{4}} .
$$

Note that, more generally, for the linear equation, the result of Proposition 2.7 holds for unbounded domains, assuming one does not have any trapped rays. In fact, for such domains, the local smoothing estimate holds ([4]), irrespective of the dimension and with an absolute constant (independent of $T$ ); a simple integration by part argument (close to the boundary) yields control of the boundary term. As such, one obtains

TheOREM 2.6. - Let $n \geq 2, \Omega \subset \mathbb{R}^{n}$ be a domain where (2.20) holds for the linear equation, and $u$ a solution to the linear equation $(2.15)(\varepsilon=0)$. Then the following estimate holds:

$$
\left\|\left|\nabla_{x}\right|^{\frac{3-n}{2}}\left(|u|^{2}\right)\right\|_{L_{t, x}^{2}} \lesssim\left\|u_{0}\right\|_{\dot{H}_{0}^{\frac{1}{4}}(\Omega)}^{2} .
$$

Now, consider the linear equation on a domain for which local smoothing does not hold. By tailoring the size of the time interval to the frequency of the solution, one may obtain an estimate with a $1 / 4$ loss of regularity.

TheOREM 2.7. - Let $\Omega \subset \mathbb{R}^{n}$ be a bounded domain, u solution to the linear equation (2.15) $(\varepsilon=0)$. Then

$$
\left\|\left|\nabla_{x}\right|^{\frac{3-n}{2}}\left(|u|^{2}\right)\right\|_{L^{2}\left([0,1] ; L^{2}(\Omega)\right)} \lesssim\left\|u_{0}\right\|_{\dot{H}_{0}^{\frac{1}{2}}(\Omega)}^{2} \cdot
$$

We remark that the boundedness of the domain is in no way essential.

REMARK 2.10. - The numerology of (2.23) is consistent with the numerology of [6] on manifolds without boundaries. By contrast, estimates from [2] have an additional $\frac{1}{3 p}$ loss, where $p$ is the time Lebesgue exponent; our example suggests better estimates than the ones which are obtained by interpolation between the $p=2$ case and the conservation of energy/mass. 


\section{Applications}

\subsection{Linear estimates on $\mathbb{R}^{n}$}

In the specific case of the linear equation $(\varepsilon=0)$, one may extend the identities of Theorems 2.1 and 2.2 through a limiting argument in the spirit of [30]. Theorem 2.4 is in fact a consequence of upcoming Theorem 3.2. We start with the 1D case, which can also be derived by an explicit computation in Fourier space, see [22]. We will use the following definition of the Fourier transform of a function $f$

$$
\hat{f}(\xi)=\int_{\mathbb{R}^{n}} e^{-i 2 \pi x \xi} f(x) d x .
$$

Theorem 3.1 (Ozawa-Tsutsumi [22]). - Let $n=1$, u, v two solutions to (2.1), (2.2) with $\varepsilon=0$, then

$$
\int_{\mathbb{R} \times \mathbb{R}}\left|\partial_{x}(u \bar{v})\right|^{2} d x d t=4 \pi \int_{\mathbb{R} \times \mathbb{R}}|\xi-\eta|\left|\hat{u}_{0}\right|^{2}(\xi)\left|\hat{v}_{0}\right|^{2}(\eta) d \xi d \eta .
$$

In higher dimensions, one has

Theorem 3.2. - Let $\omega \in \mathbb{R}^{n}$ with $|\omega|=1$, u solution to (2.15) with $\varepsilon=0$. Then, with $x=x^{\perp}+s \omega$

$$
\int_{s}\left|\partial_{s}\left(R\left(|u|^{2}\right)\right)(s, \omega)\right|^{2} d s
$$

$$
\begin{aligned}
+\int_{t} \int_{s} \int_{x \cdot \omega=s} \int_{y \cdot \omega=s} \mid u\left(x^{\perp}+s \omega\right) \partial_{s} u\left(y^{\perp}\right. & +s \omega)-\left.u\left(y^{\perp}+s \omega\right) \partial_{s} u\left(x^{\perp}+s \omega\right)\right|^{2} d x^{\perp} d y^{\perp} d s d t \\
& =4 \pi \int_{\mathbb{R}^{n} \times \mathbb{R}^{n}}|\omega \cdot(\xi-\eta)|\left|\hat{u}_{0}\right|^{2}(\xi)\left|\hat{u}_{0}\right|^{2}(\eta) d \xi d \eta .
\end{aligned}
$$

Theorem 2.1 may be used in a different direction, recovering a known bound for the linear equation (see [3]).

Proposition 3.1. - Let $u$ and $v$ be two solutions to (2.1), with $\varepsilon=0$ and data $u_{0}, v_{0}$. Assume moreover that $\operatorname{supp} \hat{u}(\xi) \subset\left\{|\xi| \leq 2^{k}\right\}$ and $\operatorname{supp} \hat{v}\left(\xi-\xi_{0}\right) \subset\left\{|\xi| \leq 2^{k}\right\}$, with $\left|\xi_{0}\right| \sim 2^{j}$ and $k \ll j$ (hence, the Fourier supports are separated and at distance roughly $2^{j}$ ). Then

$$
\|u v\|_{L_{t, x}^{2}}^{2} \lesssim 2^{(n-1) k-j}\left\|u_{0}\right\|_{L_{x}^{2}}^{2}\left\|v_{0}\right\|_{L_{x}^{2}}^{2} .
$$

\subsection{Scattering in $\mathbb{R}^{n}$}

A simple application of Theorems 2.1 and 2.2 is to recover Nakanishi's scattering result for the $H^{1}$-subcritical (and $L^{2}$-supercritical) defocusing equation. Such an alternative proof was mentioned in [12] for the cubic defocusing NLS in 3D (the authors actually proved a better result, as scattering is proved to hold for $\left.H^{s}, s>4 / 5\right)$, and done in detail for the aforementioned range in $1 \mathrm{D}$ in [11], where an a priori $L_{t, x}^{8}$ bound was derived from a four particles interaction Morawetz inequality.

TheOREM 3.3. - Let $u_{0} \in H^{1}\left(\mathbb{R}^{n}\right), n \geq 1$ and $u$ be the associated solution to (2.1) with $\varepsilon=1,1+\frac{4}{n}<p<1+\frac{4}{n-2}$. Then one has scattering and polynomial bounds on space-time norms in term of mass $M=\int\left|u_{0}\right|^{2}$ and energy $E=\int\left|\nabla_{x} u_{0}\right|^{2}+2\left|u_{0}\right|^{p+1} /(p+1)$. 
Remark 3.2. - Theorem 3.3 may also be found in [10]. In fact, the authors go beyond the $H^{1}$ theory and establish global existence and scattering for $H^{s}$ data, where $s_{p}<s<1$ and $s_{p}$ is a critical exponent for their argument. One may also consult the very recent survey [16], which encompasses all the known results, including ours, as well as extends the argument to Hartree equations.

\subsection{Existence and scattering on a 3D exterior domain}

Due to the unavailability of scale-invariant Strichartz estimates, the scattering issue is more difficult. In fact, well-posedness in the energy class is already a significantly more difficult problem, and is known to hold up to $p<3$ ([5]), $p=3$ ([18] and [1]); in these references, non sharp (non scale-invariant) Strichartz estimates are obtained and turned into the local existence result. Note that in $3 \mathrm{D}$, our estimate is better with respect to scaling (sharp estimate with a loss of a $1 / 4$ derivative) but somehow restrictive due to both the time integrability range and the derivative loss. We first deal with existence.

TheOREM 3.4. - Let $1<p<5$ and $n=3$. Let $u_{0} \in H_{0}^{1}(\Omega)$, with $\Omega$ an exterior domain where local smoothing holds, $K$ a compact set such that $\Omega^{c} \Subset K$. Then there exists a local in time solution u to (2.15) which is $C_{t}\left(H_{0}^{1}(\Omega)\right)$. Uniqueness holds in $C_{T}\left(H_{0}^{1}\right) \cap L_{T}^{4}\left(W^{\frac{3}{4}, 4}\right) \cap$ $L_{T}^{2}\left(H^{\frac{3}{2}}(K)\right) \cap L_{T}^{4}\left(L_{x}^{\infty}\left(K^{c}\right)\right)$. Moreover, when $\varepsilon=1$ (defocusing case), the solution is global in time.

When the domain $\Omega$ is star-shaped, one may use Proposition 2.9 and use the same strategy as in the $\mathbb{R}^{n}$ case to obtain scattering for the cubic equation (with some significant additional technical difficulties, due to the lack of the full set of Strichartz estimates).

Theorem 3.5. - Let $p=3$. Let $u_{0} \in H_{0}^{1}(\Omega)$, with $\Omega$ the exterior of a star-shaped domain. Then the global in time solution u to the defocusing equation (2.15) scatters in $H_{0}^{1}(\Omega)$.

\section{Proofs and further developments}

\subsection{D computation, nonlinear equation}

As a warm-up for subsequent computations, we prove Theorem 2.2 in the special case $u=v$. Let $u$ be a solution to (2.1), and let

$$
I=\int_{x>y}(x-y)|u(x)|^{2}|u(y)|^{2} d x d y .
$$

Compute the time derivative of $I$ : as we have $i \partial_{t} u+\partial_{x}^{2} u=\varepsilon|u|^{p-1} u=f$, the nonlinear part vanishes when computing

$$
\frac{d|u(x)|^{2}}{d t}=\frac{1}{i}\left(u \partial_{x}^{2} \bar{u}-\bar{u} \partial_{x}^{2} u\right)=i \partial_{x}\left(\bar{u} \partial_{x} u-u \partial_{x} \bar{u}\right)=-2 \partial_{x}\left(\operatorname{Im}\left(\bar{u} \partial_{x} u\right)\right),
$$

and we have

$$
\begin{aligned}
\partial_{t} I & =-2 \int_{x>y}(x-y)\left(\partial_{x}\left(\operatorname{Im}\left(\bar{u} \partial_{x} u\right)\right)(x)|u(y)|^{2}+\partial_{y}\left(\operatorname{Im}\left(\bar{u} \partial_{y} u\right)\right)(y)|u(x)|^{2}\right) d x d y \\
& =2\left(\int_{x>y} \operatorname{Im}\left(\bar{u} \partial_{x} u\right)(x)|u(y)|^{2}-\operatorname{Im}\left(\bar{u} \partial_{y} u\right)(y)|u(x)|^{2} d y d x\right) .
\end{aligned}
$$

$4^{\mathrm{e}}$ SÉRIE - TOME $42-2009-\mathrm{N}^{\mathrm{o}} 2$ 
Derive again in time and focus on the first term: it will be a sum of 3 terms $\left(K_{1}, K_{2}, K_{3}\right)$

$$
\begin{aligned}
K_{1} & =2 \int_{x>y} \operatorname{Im}\left(\bar{u} \partial_{x} u\right)(x) \frac{d|u(y)|^{2}}{d t} d y d x=-4 \int_{x>y} \operatorname{Im}\left(\bar{u} \partial_{x} u\right)(x) \partial_{y} \operatorname{Im}\left(\bar{u} \partial_{y} u\right)(y) d y d x \\
& =-4 \int_{x}\left(\operatorname{Im}\left(\bar{u} \partial_{x} u\right)\right)^{2}(x) d x .
\end{aligned}
$$

REMARK 4.1. - Notice for further use that when picking the second term in $\dot{I}$, it will contribute exactly another $K_{1}$ term (boundary term with opposite sign).

Now, the second term is the sum of a linear term

$$
\begin{aligned}
K_{2} & =\int_{x>y}\left(i \partial_{t} u \partial_{x} \bar{u}-i \partial_{t} \bar{u} \partial_{x} u\right)(x)|u(y)|^{2} d y d x=\int_{x>y}\left(-\partial_{x}\left(\left|\partial_{x} u\right|^{2}\right)(x)|u(y)|^{2} d y d x\right. \\
& =\int_{y}\left|\partial_{y} u\right|^{2}(y)|u(y)|^{2} d y=\int_{y}\left|\bar{u} \partial_{y} u\right|^{2}(y) d y
\end{aligned}
$$

and a nonlinear term

$$
\begin{aligned}
A_{2} & =\int_{x>y}\left(f \partial_{x} \bar{u}+\bar{f} \partial_{x} u\right)(x)|u(y)|^{2} d y d x=\int_{x>y}|u|^{p-1}(x) \partial_{x}\left(|u|^{2}\right)(x)|u(y)|^{2} d y d x \\
& =-\frac{2}{p+1} \int_{y}|u|^{p+3}(y) d y .
\end{aligned}
$$

The same remark applies for the other contribution with $x$ and $y$ reversed (so we double $K_{2}+A_{2}$ ). The next term is

$$
\begin{aligned}
K_{3} & =\int_{x>y}\left(i u \partial_{x} \partial_{t} \bar{u}-i \bar{u} \partial_{x} \partial_{t} u\right)(x)|u(y)|^{2} d y d x \\
& =\int_{x>y}-\left(i \partial_{x} u \partial_{t} \bar{u}-i \partial_{x} \bar{u} \partial_{t} u\right)(x)|u(y)|^{2} d y d x+K_{4}=K_{2}+K_{4},
\end{aligned}
$$

with $K_{4}$ being the boundary term, namely

$$
\begin{aligned}
K_{4} & =-\int_{y}\left(i u \partial_{t} \bar{u}-i \bar{u} \partial_{t} u\right)(y)|u(y)|^{2} d y=-\int_{y}\left(u \partial_{y}^{2} \bar{u}+\bar{u} \partial_{y}^{2} u\right)(y)|u(y)|^{2} d y \\
& =\int_{y} 2\left|\partial_{y} u\right|^{2}(y)|u(y)|^{2}+\left(u \partial_{y} \bar{u}+\bar{u} \partial_{y} u\right) \partial_{y}\left(|u|^{2}\right) d y=2 K_{2}+\int_{y}\left(\partial_{y}\left(|u|^{2}\right)\right)^{2} d y
\end{aligned}
$$

So that

$$
\left.K_{2}+K_{3}=4 \int_{y}\left|\partial_{y} u\right|^{2}\right)(y)|u(y)|^{2} d y+\int_{y}\left(\partial_{y}\left(|u|^{2}\right)\right)^{2} d y .
$$

The nonlinear contribution $A_{3}$ verifies the same identity, namely $A_{3}=A_{2}+A_{4}$, and $A_{4}$ is the following nonlinear boundary term:

$$
A_{4}=\int_{y}(u \bar{f}+\bar{u} f)(y)|u(y)|^{2} d y=2 \int_{y}|u(y)|^{p+3} d y
$$

and the total contribution of the nonlinear term is

$$
A=A_{2}+A_{3}=\left(2-\frac{4}{p+1}\right) \int_{y}|u|^{p+3}(y) d y .
$$


Now the claim is that the second part in $\partial_{t} I$ gives the exact same expression: $x$ and $y$ are exchanged, we have a minus sign in front, and the boundary term will be at the opposite end, switching the sign. Hence,

$$
\begin{aligned}
\frac{d^{2} I}{d t^{2}} & =2\left(K_{1}+K_{2}+K_{3}+A\right) \\
\frac{d^{2} I}{d t^{2}}-2 A & =2\left(4 \int_{x}\left(\operatorname{Re}\left(u \partial_{x} \bar{u}\right)\right)^{2}(x) d x+\int_{y}\left(\partial_{y}\left(|u|^{2}\right)\right)^{2} d y\right) \\
\frac{d^{2} I}{d t^{2}} & =4 \int_{x}\left(\partial_{x}\left(|u|^{2}\right)\right)^{2}(x) d x+4 \varepsilon \int_{y}|u|^{p+3}(y)\left(1-\frac{2}{p+1}\right) d y,
\end{aligned}
$$

which is nothing but the identity in Theorem 2.2. Notice that $I$ is a convex function whenever $\varepsilon=0,1$.

REMARK 4.2. - One may somewhat shorten the proof by introducing the density of mass $N$, the current $J$ and the (one dimensional for now!) "tensor" $T$

$$
N=|u|^{2}, J=2 \operatorname{Im}\left(\bar{u} \partial_{x} u\right), T=4\left|\partial_{x} u\right|^{2}-\Delta N+\varepsilon\left(2-\frac{4}{p+1}\right) N^{\frac{p+1}{2}},
$$

and then use local conservation laws to perform the integrations by parts

$$
\partial_{t} N+\partial_{x} J=0 \text { and } \partial_{t} J+\partial_{x} T=0 .
$$

Evidently, the relation $\partial_{t}^{2} N=\partial_{x}^{2} T$ is behind any sort of virial identity, bilinear or not, and the reader may consult [16] for a very nice survey of bilinear virial estimates, including ours, which presents the above derivation in a concise and elegant form.

\subsection{A digression on local smoothing estimates}

The 1D proof from the previous section makes crucial use of boundary terms $x=y$ arising in integrations by parts. Let us now give an elementary proof of the following well-known $1 \mathrm{D}$ estimate ([20]).

Proposition 4.3. - Let u be a solution to the linear Schrödinger equation on $\mathbb{R}$ :

$$
\sup _{x} \int_{\mathbb{R}}\left|\partial_{x} u\right|^{2}(x, t) d t=C\left\|u_{0}\right\|_{\dot{H}^{\frac{1}{2}}}^{2} .
$$

Consider $v(x)=u(x)-u(-x)$ the odd part of $u$ : $v$ still satisfies the Schrödinger equation (in fact, $v$ may be seen as a solution to the equation on $\mathbb{R}_{+}$with Dirichlet boundary condition $v(x=0)=0$ ). Multiply the equation for $v$ by $\partial_{x} \bar{v}$ and integrate between $x=\infty$ and $x=0$ :

$$
\int_{t_{1}}^{t_{2}} \int_{\infty}^{0} i\left(\partial_{t} v \partial_{x} \bar{v}-\partial_{t} \bar{v} \partial_{x} v\right)+\int_{t_{1}}^{t_{2}}\left|\partial_{x} v\right|^{2}(0)=0 .
$$

A double integration by parts in the first term yields two different types of boundary terms: time slice ones,

$$
\left|\int_{\infty}^{0} \operatorname{Im} \bar{v} \partial_{x} v d x\left(t_{1}\right)-\int_{\infty}^{0} \operatorname{Im} \bar{v} \partial_{x} v d x\left(t_{2}\right)\right| \lesssim \sup _{\left[t_{1}, t_{2}\right]}\|v\|_{\dot{H}^{\frac{1}{2}}}^{2},
$$


where one is using duality and $v(x=0)=0$. On the other hand, one has a remaining spatial boundary term,

$$
\int_{t_{1}}^{t_{2}} \operatorname{Im} \bar{v} \partial_{t} v(0)=0
$$

due to the boundary condition. Inequality in (4.3) follows trivially by translation invariance, noticing that $\partial_{x} v(0)=2 \partial_{x} u(0)$. Sending both $t_{1}$ and $t_{2}$ to $\pm \infty$ and recalling the asymptotic of the free solution would provide the equality by rewriting the momentum in term of $\hat{u}_{0}$ (see [30]).

Alternatively, one may derive this estimate by computing twice the time derivative of

$$
I=\int_{x>y}(x-y)\left|v_{y}\right|^{2}(x, t) d x, \text { with } v_{y}=u(x+y)-u(y-x) .
$$

If one goes to dimension $n$, we may instead consider the reflexion with respect to the hyperplane $x_{n}=0$ and compute

$$
I=\int_{x_{n}>y_{n}}\left(x_{n}-y_{n}\right)\left|v_{y}\right|^{2}(x, t) d x, \text { with } v_{y}(x)=u\left(x^{\prime}, x_{n}+y_{n}\right)-u\left(x^{\prime}, y_{n}-x_{n}\right) .
$$

In the computation, one may pick up additional boundary terms, namely $\int_{t}\left|\nabla^{\prime} v_{y}\right|^{2}\left(x^{\prime}, y_{n}\right) d x^{\prime} d t$, which vanish thanks to $v_{y}\left(x_{n}=y_{n}\right)=0$. Hence, we have obtained a very elementary proof of the following variant of the local smoothing effect, with no use of the Fourier transform in space or time. One, however, relies heavily on the invariances.

Proposition 4.4. - Let $u$ be a solution to the linear Schrödinger equation on $\mathbb{R}^{n}$, and $\omega$ a direction, with $x=\left(x_{\omega}^{\perp}, x_{\omega}\right)$ :

$$
\sup _{x_{\omega}} \int_{\mathbb{R} \times \mathbb{R}^{n-1}}\left|\partial_{x_{\omega}} u\right|^{2}(x, t) d x_{\omega}^{\perp} d t \lesssim\left\|u_{0}\right\|_{\dot{H}^{\frac{1}{2}}}^{2} .
$$

In view of this computation, the weight $\rho(x-y)=(x-y) \cdot \omega$ appears to be a rather natural choice in $\mathbb{R}^{n}$, when trying to average the virial on the half-space $(x-y) \cdot \omega>0$.

\subsection{Bilinear estimate on the nonlinear equation, the general case}

We now turn our attention to the general case, and prove Theorems 2.1,2.2, 2.3, 2.5 all together. We consider the equation on a domain $\Omega$, with Dirichlet boundary conditions $u_{\mid \partial \Omega}=0$. Recall that

$$
i \partial_{t}\left(|u|^{2}\right)=u \Delta \bar{u}-\bar{u} \Delta u=\nabla \cdot(u \nabla \bar{u}-\bar{u} \nabla u)=-2 i \nabla \cdot \operatorname{Im}(\bar{u} \nabla u) .
$$

Set

$$
I=\int_{\Omega \times \Omega} \rho(x-y)|u|^{2}(x)|v|^{2}(y) d x d y
$$

We compute

$$
\begin{aligned}
\partial_{t} I & =-2 \int \rho\left(|v|^{2} \nabla \cdot \operatorname{Im}(\bar{u} \nabla u)+|u|^{2} \nabla \cdot \operatorname{Im}(\bar{v} \nabla v)\right) \\
& =2 \int \nabla_{x} \rho \cdot\left(|v|^{2}(y) \operatorname{Im}(\bar{u} \nabla u)(x)-|u|^{2}(x) \operatorname{Im}(\bar{v} \nabla v)(y)\right) d x d y,
\end{aligned}
$$

where there is no boundary term when applying Stokes, as there is always a factor of $u$ or $v$ to cancel such a term due to the Dirichlet condition, and we used $\nabla_{x} \rho=-\nabla_{y} \rho$. 
Now, we compute $\partial_{t}^{2} I=J_{x}+J_{y}+J_{x y}$ depending on where the time derivative lands (with obvious notations). We have

$$
J_{x}=\int|v|^{2}(y) \nabla_{x} \rho \cdot \partial_{t}\left(\frac{\bar{u} \nabla u-u \nabla \bar{u}}{i}\right)(x) d x d y .
$$

Now

$$
\begin{aligned}
\partial_{t}\left(\frac{\bar{u} \nabla u-u \nabla \bar{u}}{i}\right)= & \left(-\Delta u+\varepsilon|u|^{p-1} u\right) \nabla \bar{u}+\left(-\Delta \bar{u}+\varepsilon|u|^{p-1} \bar{u}\right) \nabla u \\
& -\left[u \nabla\left(-\Delta \bar{u}+\varepsilon|u|^{p-1} \bar{u}\right)+\bar{u} \nabla\left(-\Delta u+\varepsilon|u|^{p-1} u\right)\right] \\
= & -\Delta u \nabla \bar{u}-\Delta \bar{u} \nabla u+u \nabla \Delta \bar{u}+\bar{u} \nabla \Delta u-\varepsilon|u|^{2} \nabla\left(|u|^{p-1}\right) .
\end{aligned}
$$

Back to $J_{x}$, we call $K_{1}, K_{2}$ the bilinear and nonlinear terms coming from the above formula. We use Einstein convention for summation:

$$
\begin{aligned}
K_{1} & =\int|v|^{2} \partial_{i} \rho\left(-\partial_{k} \partial^{k} u \partial^{i} \bar{u}-\partial_{k} \partial^{k} \bar{u} \partial^{i} u\right)+\int|v|^{2} \partial_{i} \rho\left(u \partial^{i} \partial_{k} \partial^{k} \bar{u}+\bar{u} \partial^{i} \partial_{k} \partial^{k} u\right) \\
& =K_{11}+K_{12}
\end{aligned}
$$

We have $(n(x)$ being the outgoing normal vector at $x \in \partial \Omega)$

$$
\begin{aligned}
K_{11}= & \int|v|^{2}(y) \nabla_{x} \rho(x-y) \cdot\left(\nabla_{x} \bar{u}\left(-\nabla_{x} \cdot \nabla_{x} u\right)+\nabla_{x} u\left(-\nabla_{x} \cdot \nabla_{x} \bar{u}\right)\right)(x) \\
= & -\int|v|^{2}(y)\left(\nabla_{x} \rho \cdot \nabla_{x} \bar{u} \nabla_{x} u \cdot n(x)+\nabla_{x} \rho \cdot \nabla_{x} u \nabla_{x} \bar{u} \cdot n(x)\right) d S_{x} \\
& +\int|v|^{2}(y)\left(\nabla _ { x } u \cdot \nabla _ { x } \left(\nabla_{x} \rho \cdot \nabla_{x} \bar{u}+\nabla_{x} \bar{u} \cdot \nabla_{x}\left(\nabla_{x} \rho \cdot \nabla_{x} u\right)(x)\right.\right. \\
= & -2 \int|v|^{2}(y) \partial_{n} \rho(x-y)\left|\partial_{n} u\right|^{2}(x) d S_{x} d y \\
& +2 \int|v|^{2} \partial_{i} \partial^{k} \rho \partial_{k} u \partial^{i} \bar{u} \text { (recall the Hessian is symmetric) } \\
& +\int|v|^{2} \partial_{i} \rho\left(\partial_{k} u \partial^{i} \partial^{k} \bar{u}+\partial_{k} \bar{u} \partial^{k} \partial^{i} u\right)
\end{aligned}
$$

where we used the Dirichlet condition in the boundary term and expanded the remaining terms. On the other hand, as all boundary terms cancel due to the Dirichlet condition,

$$
K_{12}=-\int|v|^{2} \partial_{i} \partial^{k} \rho\left(u \partial^{i} \partial_{k} \bar{u}+\bar{u} \partial^{i} \partial_{k} u\right)-\int|v|^{2} \partial_{i} \rho\left(\partial^{k} u \partial^{i} \partial_{k} \bar{u}+\partial^{k} \bar{u} \partial^{i} \partial_{k} u\right) .
$$

Summing $K_{11}$ and $K_{12}$, their respective last terms cancel each other. Integrate the first term in $K_{12}$ with respect to $\partial^{i}$, there is (again) no boundary term, and finally

$$
\begin{aligned}
K_{1}=4 \int|v|^{2}(y) \partial_{i} \partial^{k} \rho(x-y) \partial_{k} u \partial^{i} \bar{u}(x)+ & \int|v|^{2}(y) \partial^{i} \partial_{i} \partial^{k} \rho(x-y) \partial_{k}\left(|u|^{2}\right)(x) d x d y \\
& -2 \int|v|^{2}(y) \partial_{n} \rho(x-y)\left|\partial_{n} u\right|^{2}(x) d S_{x} d y
\end{aligned}
$$


However, $\partial_{x_{i}}^{2} \partial_{x_{k}} \rho=-\partial_{x_{i}}^{2} \partial_{y_{k}} \rho=-\partial_{x_{i}} \partial_{x_{k}} \partial_{y_{i}} \rho$, so that one last integration by parts yields, denoting by $H_{\rho}$ the Hessian of $\rho$,

$$
\begin{aligned}
K_{1}= & 4 \int|v|^{2}(y) H_{\rho}(x-y)\left(\nabla_{x} u(x), \nabla_{x} \bar{u}(x)\right) d x d y \\
& +\int H_{\rho}(x-y)\left(\nabla_{x}\left(|u|^{2}\right)(x), \nabla_{y}\left(|v|^{2}\right)(y)\right) d x d y \\
& -2 \int|v|^{2}(y) \partial_{n} \rho(x-y)\left|\partial_{n} u\right|^{2}(x) d S_{x} d y,
\end{aligned}
$$

given that the integration by parts in $y$ does not have a boundary term either, and where we may freely replace the second term using the identity

$\int H_{\rho}(x-y)\left(\nabla_{x}\left(|u|^{2}\right)(x), \nabla_{y}\left(|v|^{2}\right)(y)\right) d x d y=\int \Delta \rho(x-y) \nabla_{x}\left(|u|^{2}\right)(x) \cdot \nabla_{y}\left(|v|^{2}\right)(y) d x d y$.

Now, we go back to the nonlinear term $K_{2}$ :

$$
\begin{aligned}
K_{2} & =-\int|v|^{2}(y) \nabla_{x} \rho \cdot \varepsilon\left(|u|^{p-1}\right)^{\frac{2}{p-1}} \nabla\left(|u|^{p-1}\right) d x d y \\
& =-\varepsilon \int|v|^{2}(y) \nabla_{x} \rho \cdot \nabla\left(|u|^{p+1}\right) \frac{1}{\frac{2}{p-1}+1} d x d y \\
& =\varepsilon \int|v|^{2}(y)\left(\Delta_{x} \rho\right)(x-y)|u|^{p+1}(x) \frac{p-1}{p+1} d x d y,
\end{aligned}
$$

performing one more integration by parts (with no boundary term). Assuming that $\rho(x-y)=\rho(y-x)$, the second term $J_{y}$ is exactly $J_{x}$ by symmetry, up to permutation of $u$ and $v$. We are left with

$$
J_{x y}=J_{1}+J_{2}
$$

where again by symmetry both terms are equal and

$$
\begin{aligned}
J_{1} & =2 \int \operatorname{Im}(\bar{u} \nabla u)(x) \cdot \nabla_{x} \rho \partial_{t}\left(|v|^{2}(y)\right) d x d y \\
& =4 \int \operatorname{Im}(\bar{u} \nabla u)(x) \cdot \nabla_{x} \rho \nabla_{y} \operatorname{Im}(\bar{v} \nabla v(y)) d x d y
\end{aligned}
$$

and using again $\nabla_{y} \rho=-\nabla_{x} \rho$, we integrate by parts in $y$ (with no boundary term)

$$
J_{1}=-4 \int H_{\rho}(x-y)(\operatorname{Im}(\bar{u} \nabla u)(x), \operatorname{Im}(\bar{v} \nabla v)(y)) d x d y .
$$

Finally,

$$
\begin{aligned}
\partial_{t}^{2} I= & 4 \int\left(|v|^{2}(y) H_{\rho}(x-y)(\nabla u(x), \nabla \bar{u}(x))+|u|^{2}(x) H_{\rho}(x-y)(\nabla v(y), \nabla \bar{v}(y))\right) d x d y \\
& +2 \int\left(H_{\rho}(x-y)\left(\nabla\left(|u|^{2}\right)(x), \nabla\left(|v|^{2}\right)(y)\right)-4 H_{\rho}(x-y)(\operatorname{Im}(\bar{u} \nabla u)(x), \operatorname{Im}(\bar{v} \nabla v)(y))\right) d x d y \\
& +\varepsilon\left(1-\frac{2}{p+1}\right) \int\left(|v|^{2}(y)(\Delta \rho)(x-y)|u|^{p+1}(x)+|u|^{2}(x)\left(\Delta_{x} \rho\right)(x-y)|v|^{p+1}(y)\right) d x d y \\
& -2 \int|v|^{2}(y) \partial_{n} \rho(x-y)\left|\partial_{n} u\right|^{2}(x) d S_{x} d y-2 \int|u|^{2}(x) \partial_{n} \rho(x-y)\left|\partial_{n} v\right|^{2}(y) d S_{y} d x
\end{aligned}
$$

By definition, $H_{\rho}$ is symmetric. Then one may diagonalize and be left with just one direction (or, more accurately, a diagonalized matrix). Discarding a factor 2 and the eigenvalue 
$\lambda(x-y)$, we set (where $\partial$ denotes derivation in the direction of the eigenvector associated to $\lambda$ )

$$
\begin{aligned}
\Gamma= & 2|v|^{2}(y)|\partial u|^{2}(x)+2|u|^{2}(x)|\partial v|^{2}(y) \\
& +(v \partial \bar{v}+\bar{v} \partial v)(y)(u \partial \bar{u}+\bar{u} \partial u)(x)-(v \partial \bar{v}-\bar{v} \partial v)(y)(\bar{u} \partial u-u \partial \bar{u})(x) .
\end{aligned}
$$

Expanding the last two terms and canceling out, we get

$$
\begin{aligned}
\Gamma & =2|v|^{2}(y)|\partial u|^{2}(x)+2|u|^{2}(x)|\partial v|^{2}(y)+2 v \partial \bar{v}(y) u \partial \bar{u}(x)+2 \bar{v} \partial v(y) \bar{u} \partial u(x) \\
& =2|\bar{v}(y) \partial u(x)+u(x) \partial \bar{v}(y)|^{2} .
\end{aligned}
$$

Now, one may rewrite $\Gamma$ in a different way, by taking advantage of the identity

$$
|v(y) \partial u(x)-u(x) \partial v(y)|^{2}+\partial\left(|v|^{2}\right)(y) \partial\left(|u|^{2}\right)(x)=|\bar{v}(y) \partial u(x)+u(x) \partial \bar{v}(y)|^{2} .
$$

This achieves the proof of the first part of Theorem 2.3, namely (2.12) and (2.13). Finally, in the special case $n=1$ we obtain Theorem 2.2. A straightforward generalization of the $n=1$ case will follow by setting $\rho=|(x-y) \cdot \omega|$ with $\omega \in \mathbb{S}^{n}$ : we will obtain Theorem 2.1 and Theorem 2.5.

REMARK 4.5. - Notice that if $u=v$ and $x=y$, one recovers the same identity, with $\Gamma=2\left(\partial\left(|u|^{2}\right)\right)^{2}$, with both expressions, which is consistent with our previous 1D computation.

Set $\rho=\left|x_{n}-y_{n}\right|$ for convenience, and let us focus on the linear equation in $\mathbb{R}^{n}$; we have obtained, discarding a positive term, the inequality

$$
\left.\int_{-T}^{T} \int_{x_{n}}\left(\partial_{n}\left(\int_{x^{\prime}}|u|^{2}\left(x^{\prime}, x_{n}, t\right) d x^{\prime}\right)\right)^{2} d x_{n} d t \lesssim \int_{x_{n}<y_{n}} \operatorname{Im} \bar{u} \partial_{n} u(x)|u|^{2}(y) d x d y\right|_{-T} ^{T} .
$$

Proceeding exactly as in [30], one may send $T \rightarrow+\infty$ and recover an exact formula for the right-hand side. Recall the following asymptotic formula for the solution $U(t, z)$ to the linear equation $i \partial_{t} U+\Delta U=0$ with data $U_{0}$, and $z \in \mathbb{R}^{m}$, which follows directly from the explicit representation as a convolution by the Gaussian kernel $(4 \pi i t)^{-m / 2} \exp \left(i|z|^{2} / 4 t\right)$ :

$$
\lim _{t \rightarrow \pm \infty}\left\|U(t, z)-\frac{e^{ \pm i \frac{|z|^{2}}{4 t}}}{(4 \pi i t)^{m / 2}} \hat{U}_{0}\left( \pm \frac{z}{4 \pi|t|}\right)\right\|_{L^{2}\left(\mathbb{R}^{m}\right)}=0 .
$$

By using (4.9) we get

$$
\lim _{t \rightarrow+\infty}\|U(t, z)-V(t, z)\|_{L^{2}\left(\mathbb{R}^{m}\right)}=0
$$

where $V(t, z):=e^{-i m \pi / 4} \frac{e^{i \frac{|z|^{2}}{4 t}}}{(4 \pi t)^{m / 2}} \hat{U}_{0}\left(\frac{z}{t}\right)$. On the other hand, for any direction $s, \partial_{s} U$ is also a solution, hence

$$
\lim _{t \rightarrow+\infty}\left\|\partial_{s} U(t, z)-W_{s}(t, z)\right\|_{L^{2}\left(\mathbb{R}^{m}\right)}=0
$$

where

We easily deduce

$$
W_{s}(t, z):=e^{-i m \pi / 4} \frac{e^{i \frac{|z|^{2}}{4 t}}}{(4 \pi t)^{m / 2}} i \frac{s}{2 t} \hat{U}_{0}\left(\frac{z}{4 \pi t}\right) .
$$

$$
\lim _{t \rightarrow+\infty} \int_{\mathbb{R}^{m}}\left[\bar{U}(t, z) \partial_{s} U(t, z)-\bar{V}(t, z) W_{s}(t, z)\right] \phi(s) d z=0
$$


for any $\phi \in L^{\infty}$. Then if $\phi(s)=\partial_{s}|s|$,

$$
\begin{aligned}
\lim _{t \rightarrow+\infty} \operatorname{Im} \int_{\mathbb{R}^{m}} \bar{U}(t, z) \phi(s) \partial_{s} U(t, z) d z & =\lim _{t \rightarrow+\infty} \operatorname{Im} \int_{\mathbb{R}^{m}} \bar{V}(t, z) W_{s}(t, z) \phi(s) d z \\
& =\lim _{t \rightarrow+\infty}(4 \pi t)^{-m} \int_{\mathbb{R}^{m}} \frac{s}{2 t}\left|\hat{U}_{0}\left(\frac{z}{4 \pi t}\right)\right|^{2} \phi(s) d z \\
& =4 \pi \int_{\mathbb{R}^{m}} \frac{|s|}{2}\left|\hat{U}_{0}(z)\right|^{2} d z .
\end{aligned}
$$

Let $u$ be a solution of the linear equation in $\mathbb{R}^{n}$. We proceed with a tensor product solution and set $U(t, z)=u(t, x) u(t, y)$ with $z=(x, y), m=2 n$, and pick the direction $s=x_{n}-y_{n}$. Then the limit when $T \rightarrow+\infty$ of the right-hand side in (4.8) will be a multiple of $\int\left|x_{n}-y_{n}\right|\left|\hat{u}_{0}\right|^{2}(x)\left|\hat{u}_{0}\right|^{2}(y) d x d y$. Hence we have obtained

$$
\int_{t} \int_{x_{n}}\left(\partial_{n}\left(\int_{x^{\prime}}|u|^{2}\left(x^{\prime}, x_{n}, t\right) d x^{\prime}\right)\right)^{2} d x_{n} d t \lesssim \int\left|\xi_{n}-\eta_{n}\right|\left|\hat{u}_{0}\right|^{2}(\xi)\left|\hat{u}_{0}\right|^{2}(\eta) d \xi d \eta .
$$

Up to the upcoming introduction of the Radon transform, this is exactly Theorem 2.4 but with $u=v$. Applying this estimate to $u+v$ and $u+i v$ allows to control both $\left|\partial_{n}(\operatorname{Re}(u \bar{v}))\right|$ and $\left|\partial_{n}(\operatorname{Im}(u \bar{v}))\right|$, and applying Cauchy-Schwarz repeatedly on the right-hand side, we obtain Theorem 2.4. On the other hand, in the special case $n=1$, one does not need to set $u=v$ and we obtain Theorem 3.1. Finally, if we retain the discarded term in (4.8) and keep $u=v$, we obtain Theorem 3.2.

Dilating $u$ and $v$ in opposite way and optimizing allows us to replace the right-hand side by $\left\|u_{0}\right\|_{L_{x}^{2}}\left\|v_{0}\right\|_{\dot{H}^{\frac{1}{2}}}+\left\|v_{0}\right\|_{L_{x}^{2}}\left\|u_{0}\right\|_{\dot{H}^{\frac{1}{2}}}$, up to Proposition 2.2 whose proof we postpone for the moment.

Now we reduce the directional estimate we obtained to a generic one by introducing the Radon transform. By rotation, one may replace $x_{n}$ by the coordinate along any direction $\omega$, so that if $R(f)(s, \omega)$ is the Radon transform of a function $f$, namely

$$
R(f)(s, \omega)=\int_{x \cdot \omega=s} f d \mu_{s, \omega},
$$

where $\mu_{s, \omega}$ is the induced measure on the hyperplane $x \cdot \omega=s$, the previous estimate can be recast as

$$
\sup _{\omega} \int_{t} \int_{s}\left|\partial_{s}(R(u \bar{v}))(s, \omega)\right|^{2} d s d t \lesssim\left\|u_{0}\right\|_{L_{x}^{2}}^{2}\left\|v_{0}\right\|_{\dot{H}^{\frac{1}{2}}}^{2}+\left\|v_{0}\right\|_{L_{x}^{2}}^{2}\left\|u_{0}\right\|_{\dot{H}^{\frac{1}{2}}}^{2} \cdot
$$

Replacing the $L_{\omega}^{\infty}$ by $L_{\omega}^{2}$ and using that

$$
\left\|\left|\partial_{s}\right|^{\frac{n-1}{2}} R(f)\right\|_{L^{2}}=\|f\|_{L^{2}}
$$

one recovers known bounds on the linear equation for $n=2$ and $n=3$ :

- if $n=2$,

$$
\int_{t_{1}}^{t_{2}}\left\||\nabla|^{\frac{1}{2}}(u \bar{v})\right\|_{L^{2}}^{2} d t \lesssim\left\|u_{0}\right\|_{L_{x}^{2}}^{2}\left\|v_{0}\right\|_{\dot{H}^{\frac{1}{2}}}^{2}+\left\|v_{0}\right\|_{L_{x}^{2}}^{2}\left\|u_{0}\right\|_{\dot{H}^{\frac{1}{2}}}^{2} \cdot
$$

One may get an $L_{t}^{4}\left(L_{x}^{8}\right)$ bound for $\dot{H}^{\frac{1}{4}}$ data, but fails short of getting the usual $L_{t, x}^{4}$ bound. We will recover this bound through a refined analysis using the Radon bound in a more efficient way. 
- if $n=3$

$$
\int_{t_{1}}^{t_{2}}\|(u \bar{v})\|_{L^{2}}^{2} d t \lesssim\left\|u_{0}\right\|_{L_{x}^{2}}^{2}\left\|v_{0}\right\|_{\dot{H}^{\frac{1}{2}}}^{2}+\left\|v_{0}\right\|_{L_{x}^{2}}^{2}\left\|u_{0}\right\|_{\dot{H}^{\frac{1}{2}}}^{2}
$$

which is a (linear) variation on the original $L_{t, x}^{4}$ Morawetz interaction estimate from [12].

Next, one would like to take advantage of the $L_{\omega}^{\infty}$ bound. Consider the situation where $v$ is frequency localized in a (small) ball $|\xi| \lesssim 2^{k}$ and $u$ is frequency localized in a ball of the same size but which is included in the annulus $|\xi| \sim 2^{j}$, with $k \ll j$. The Fourier transform of $u \bar{v}$ has roughly the same frequency localization as $u$, hence it is supported in a ball of size $2^{k}$ and in an angular sector of (angular) width $2^{(n-1)(k-j)}$ (the volume of the $(n-1)$ dimensional cap which is the intersection of the angular sector and the sphere of radius 1$)$. As the Fourier transform of $R(f)$ is connected with $\hat{f}$ by the following formula

$$
g(\rho, \omega)=\mathcal{F}_{s \rightarrow \rho}(R f(., \omega))[\rho]=\hat{f}(\rho \omega) \forall \omega \in \mathbb{S}^{n-1},
$$

we will have, for such $f=u \bar{v}$, by Plancherel,

$$
\int_{\rho, \omega} \rho^{3-n}|\hat{f}(\rho \omega)|^{2} \rho^{n-1} d \rho d \omega \lesssim 2^{(n-1)(k-j)} \sup _{\omega} \int_{\rho}|\rho g(\rho, \omega)|^{2} d \rho
$$

which translates into

$$
\left\||\nabla|^{\frac{1}{2}}(u \bar{v})\right\|_{L_{t, x}^{2}}^{2} \lesssim 2^{(n-1) k-j}\left(\left\|u_{0}\right\|_{\dot{H}^{\frac{1}{2}}}^{2}\left\|v_{0}\right\|_{L^{2}}^{2}+\left\|v_{0}\right\|_{\dot{H}^{\frac{1}{2}}}^{2}\left\|u_{0}\right\|_{L^{2}}^{2}\right),
$$

and due to the frequency localization, one may remove the half-derivative on both sides; the complex conjugate is now irrelevant, and we get (3.3). For example Bourgain's original estimate for $n=2([3])$ reads

$$
\|u v\|_{L_{t, x}^{2}}^{2} \lesssim 2^{k-j}\left\|u_{0}\right\|_{L^{2}}^{2}\left\|v_{0}\right\|_{L^{2}}^{2}
$$

By a Galilean transform, one may shift both factors by any $\xi_{0}$ in frequency space, as both norms on the right and the left are galilean invariant. Thus, we obtain that for $u, v$ such that their Fourier supports are in balls of radius $2^{k}$ which are $2^{j}$ apart,

$$
\|u v\|_{L_{t, x}^{2}}^{2} \lesssim 2^{k-j}\left\|u_{0}\right\|_{L^{2}}^{2}\left\|v_{0}\right\|_{L^{2}}^{2} .
$$

Assuming only (4.18), one may then recover the usual $L_{t, x}^{4}$ bound by the usual Whitney decomposition trick, see [27]. However, we may derive it directly: consider

$$
|u|^{2}=\sum_{j} S_{j-2} \bar{u} \Delta_{j} u+\sum_{j} S_{j-2} \bar{u} \Delta_{j} u+\sum_{\left|j-j^{\prime}\right| \leq 1} \Delta_{j^{\prime}} \bar{u} \Delta_{j} u,
$$

the usual paraproduct decomposition. On both paraproduct terms, we take advantage of the frequency separation; applying (4.18) provides the $L_{t, x}^{4}$ bound. On the reminder term, we have to consider (abusing notations by reducing the sum to the diagonal one)

$$
\Delta_{k}\left(\sum_{k \lesssim j} \Delta_{j} u \Delta_{j} \bar{u}\right) .
$$

If $j \gg k$, then only opposite balls of radius $2^{k}$ (and at distance $2^{j}$ from $\xi=0$ ) contribute, and again we may use (4.18) and sum in $j$. When $k \sim j$, either the two supports are separated and (4.18) will do, or the supports are the same (splitting in a finite number of smaller balls if 
necessary), but then they do not overlap the origin in $\xi$ : one may go back to (4.14) and take advantage of the support condition to get rid of half a derivative.

Let us go back to the nonlinear equation: our choice of $\rho(x-y)=|\omega \cdot(x-y)|$ in Theorem 2.3 , together with the definition of the Radon transform, immediately yields Theorems 2.1 and 2.5, as the former is a particular case of the latter.

We now prove Proposition 2.2, starting with (2.8): but we almost did in Section 4.2. At fixed $y_{n}$,

$$
\left|\int_{x_{n}<y_{n}} \operatorname{Im}\left(\bar{u}(x)-\bar{u}\left(x^{\prime}, y_{n}\right)\right) \partial_{n} u(x) d x\right| \lesssim\|u\|_{\dot{H}^{\frac{1}{2}}}^{2},
$$

using duality in $\dot{H}_{x}^{\frac{1}{2}}\left(\mathbb{R}_{+}^{n}\right)$, as $u(x)-u\left(x^{\prime}, y_{n}\right) \in \dot{H}_{x}^{\frac{1}{2}}\left(\mathbb{R}_{+}^{n}\right)$. Then one may rewrite,

$$
\begin{aligned}
2 i \int_{x_{n}<y_{n}}|u|^{2}(y) \operatorname{Im} \bar{u}(x) \partial_{n} u(x) d x d y= & \int_{x_{n}<y_{n}}|u|^{2}(y)\left(\left(\bar{u}(x)-\bar{u}\left(x^{\prime}, y_{n}\right)\right) \partial_{n} u(x)\right. \\
& \left.-\left(u(x)-u\left(x^{\prime}, y_{n}\right)\right) \partial_{n} \bar{u}(x)\right) d x d y
\end{aligned}
$$

as

$$
\int_{x_{n}<y_{n}} \partial_{n} u(x) d x_{n}=u\left(x^{\prime}, y_{n}\right) \Longrightarrow \int_{x_{n}<y_{n}} \bar{u}\left(x^{\prime}, y_{n}\right) \partial_{n} u(x) d x=\int_{x^{\prime}}|u|^{2}\left(x^{\prime}, y_{n}\right) d x^{\prime}
$$

From there (2.8) easily follows.

We proceed with (2.9), which follows from averaging the Radon transform over directions $\omega$ in $L_{\omega}^{2}$ in (4.13): the linear part we already obtained; now both $R\left(|u|^{2}\right)$ and $R\left(|u|^{p+1}\right)$ are positive, we immediately have by Cauchy-Schwarz

$$
\left|R\left(|u|^{\frac{p+3}{2}}\right)\right|^{2} \lesssim R\left(|u|^{2}\right) R\left(|u|^{p+1}\right) .
$$

Discarding a positive term in the left-hand side of (2.19), we finally obtain (2.9) which ends the proof.

\section{Local smoothing and control of the trace for NLS on a domain}

We now prove Proposition 2.7. Let us stress, once again, that for the linear equation, (2.20) holds on any non-trapping domain for any dimension (see [4]). Hence, the purpose of this section is to provide a simple integration by parts proof when $n \geq 3$, which equally applies to the nonlinear defocusing equation. Let us consider again

$$
i \partial_{t} u+\Delta u-\varepsilon|u|^{p-1} u=0
$$

where $\Delta$ is the Laplacian with Dirichlet boundary condition $u_{\mid \partial \Omega}=0$, and $\Omega$ is the exterior of a star-shaped body with smooth boundary.

First, the virial identity (the following computation is standard and we provide it for completeness): let us denote

$$
M_{h}(t)=\int_{\Omega}|u|^{2}(x, t) h(x) d x
$$

where $h$ is any smooth real-valued function on $\Omega$. Then compute (recalling (4.5))

$$
\frac{d}{d t} M_{h}(t)=-2 \operatorname{Im} \int h \nabla \cdot(\bar{u} \nabla u)=2 \operatorname{Im} \int \bar{u} \nabla u \cdot \nabla h,
$$


where we used the Dirichlet boundary condition when integrating by parts. Now,

$$
\begin{aligned}
\frac{d^{2}}{d t^{2}} M_{h}(t)= & 2 \operatorname{Im} \int\left(\partial_{t} \bar{u} \nabla u+\bar{u} \nabla \partial_{t} u\right) \cdot \nabla h=-2 \operatorname{Im} \int \partial_{t} u(2 \nabla \bar{u} \cdot \nabla h+\bar{u} \Delta h) \\
= & -2 \operatorname{Re} \int\left(\Delta u-\varepsilon|u|^{p-1} u\right)(2 \nabla \bar{u} \cdot \nabla h+\bar{u} \Delta h) \\
= & -4 \operatorname{Re} \int \Delta u \nabla \bar{u} \cdot \nabla h+2 \int|\nabla u|^{2} \Delta h+2 \operatorname{Re} \int \bar{u} \nabla u \nabla \Delta h \\
& +2 \int \varepsilon|u|^{p-1} \nabla\left(|u|^{2}\right) \nabla h+2 \int \varepsilon|u|^{p+1} \Delta h \\
= & -4 \operatorname{Re} \int \Delta u \nabla \bar{u} \cdot \nabla h+2 \int|\nabla u|^{2} \Delta h-\int|u|^{2} \Delta^{2} h+\int 2 \varepsilon\left(1-\frac{2}{p+1}\right)|u|^{p+1} \Delta h .
\end{aligned}
$$

Integrating by parts again,

$$
\int \Delta u \nabla \bar{u} \cdot \nabla h=\int_{\partial \Omega} \nabla \bar{u} \cdot \nabla h \partial_{n} u-\int \nabla(\nabla \bar{u} \cdot \nabla h) \cdot \nabla u,
$$

and, as $u_{\partial \Omega}=0$ implies $\partial_{\tau} u_{\partial \Omega}=0$,

$$
\begin{aligned}
2 \operatorname{Re} \int \Delta u \nabla \bar{u} \cdot \nabla h & =2 \int_{\partial \Omega}\left(\partial_{n} h\right)\left|\partial_{n} u\right|^{2}-\int \nabla h \cdot \nabla\left(|\nabla u|^{2}\right)-2 \int \operatorname{Hess}(h)(\nabla u, \nabla \bar{u}) \\
& =\int_{\partial \Omega}\left(\partial_{n} h\right)\left|\partial_{n} u\right|^{2}+\int|\nabla u|^{2} \Delta h-2 \int \operatorname{Hess}(h)(\nabla u, \nabla \bar{u})
\end{aligned}
$$

and finally we obtained

$$
\frac{d^{2}}{d t^{2}} M_{h}(t)=-\int|u|^{2} \Delta^{2} h+2 \varepsilon\left(1-\frac{2}{p+1}\right) \int|u|^{p+1} \Delta h-2 \int_{\partial \Omega}\left(\partial_{n} h\right)\left|\partial_{n} u\right|^{2}+4 \int \operatorname{Hess}(h)(\nabla u, \nabla \bar{u})
$$

where we can switch sign for the boundary term if we integrate with the inner normal of the domain (outer normal of the obstacle!), retaining the same notation $\partial_{n}$ :

$$
\frac{d^{2}}{d t^{2}} M_{h}(t)=-\int|u|^{2} \Delta^{2} h+2 \varepsilon \frac{p-1}{p+1}|u|^{p+1} \Delta h+2 \int_{\partial\left(\mathbb{R}^{n} \backslash \Omega\right)}\left(\partial_{n} h\right)\left|\partial_{n} u\right|^{2}+4 \int \operatorname{Hess}(h)(\nabla u, \nabla \bar{u}) .
$$

One immediately infers that if $\varepsilon=0,1$, one controls all terms on the right-hand side of (5.2), provided $h$ is chosen to be $h(x)=\sqrt{1+|x|^{2}}$ where the origin $O$ is such that the obstacle is star-shaped with respect to $O$ : one has

$$
\Delta h=\frac{n-1}{h(x)}+\frac{1}{h(x)^{3}}, \quad-\Delta^{2} h=\frac{(n-1)(n-3)}{h(x)^{3}}+\frac{6(n-3)}{h(x)^{5}}+\frac{15}{h(x)^{7}}, \text { and } n(x) \cdot x \geq 0 .
$$

However, the boundary term may be zero at points where the outgoing ray is tangent. We will have to proceed in two steps: from the (strict) positivity of the Hessian, we get

$$
\frac{|\nabla u|^{2}}{h(x)^{3}} \lesssim \operatorname{Hess}(h)(\nabla u, \nabla \bar{u}), \text { as } \operatorname{Hess}(h)=\frac{1}{h} I d-\frac{1}{h^{3}}\left(x_{i} x_{j}\right)_{i j},
$$

which implies the local smoothing part in Proposition 2.7. Better yet, in a compact region $K$ close to the boundary, we obtain

$$
\int_{0}^{T} \int_{K}|\nabla u|^{2} d x d t+\int_{0}^{T} \int_{K}|u|^{p+1} d x d t \lesssim \sup _{[0, T]}\|u\|_{\dot{H}^{\frac{1}{2}}}^{2} .
$$


REMARK 5.1. - When $n=2$, the $\Delta^{2} h$ term has the wrong sign: one can only write (without any attempt to optimize!)

$$
\left.\int_{0}^{T}\left|\int\right| u\right|^{2} \Delta^{2} h \mid \lesssim C(\Omega) T\left\|u_{0}\right\|_{L^{2}(\Omega)}^{2}
$$

It remains to control the boundary term in Proposition 2.9. Assume for the remaining part of the proof that $\Omega$ is either the exterior of the star-shaped domain if $\varepsilon=1$, or the exterior of a non trapping domain, or a bounded domain (boundedness is not essential but allows covering of the boundary by a finite number of compact patches). Pick a part of the boundary $P$ where one has local coordinates such that the normal is a coordinate, and define $h(x)=$ $d(x, \partial \Omega) \phi(x)$ where $\phi$ is a smooth cut-off to this coordinate patch, such that on a strip close to the boundary $\phi$ only depends on the tangential variables. Hence $\partial_{n} h \geq 0$ on the boundary part of the patch, and is actually 1 on a smaller subset $Q \subset P$, and we control

$$
\int_{Q}\left|\partial_{n} u\right|^{2} \leq \int_{P}\left|\partial_{n} u\right|^{2}
$$

Now, as $M_{h} \lesssim\|u\|_{\dot{H}^{\frac{1}{2}}}^{2} \lesssim\|u\|_{L^{2}}\|u\|_{\dot{H}^{1}}$, we get using (5.2) with this $h$ (for which the Hessian, Laplacian and bilaplacian do not have a sign over the whole domain of integration), with $S$ a (compact) strip close to the boundary,

$$
\int_{0}^{T} \int_{Q}\left|\partial_{n} u\right|^{2} \lesssim \int_{0}^{T}\|u\|_{H^{1}(S)}^{2} d s+\sup _{[0, T]}\|u\|_{\dot{H}^{\frac{1}{2}}}^{2} ;
$$

indeed,when $\varepsilon=0,1$ we control the lower order terms by the gradient of $u$, and for $\varepsilon=1$ we control the nonlinear term using (5.3). Patching together a finite number of local coordinates patches, we control the entire boundary term,

$$
\int_{0}^{T} \int_{\partial \Omega}\left|\partial_{n} u\right|^{2} \lesssim \int_{0}^{T}\|u\|_{H^{1}(S)}^{2} d s+\sup _{[0, T]}\|u\|_{\dot{H}^{\frac{1}{2}}}^{2} \cdot
$$

If we are on the exterior of a star-shaped domain in the nonlinear case, from (5.3), Proposition 2.7 and Theorem 2.5, we immediately deduce Proposition 2.9.

Now, on the exterior of a compact set with no trapped rays, local smoothing holds ([4]) and we obtain a global in time control

$$
\int_{0}^{T} \int_{\partial \Omega}\left|\partial_{n} u\right|^{2} \lesssim \sup _{[0, T]}\|u\|_{\dot{H}^{\frac{1}{2}}}^{2} \lesssim\left\|u_{0}\right\|_{L^{2}}\left\|u_{0}\right\|_{\dot{H}^{1}} .
$$

Combining this with Theorem 2.5, we deduce Theorem 2.6 in the non star-shaped case, for the linear equation, provided we can replace the right-hand side $\left\|u_{0}\right\|_{L_{x}^{2}}^{2}\left\|u_{0}\right\|_{\dot{H}^{\frac{1}{2}}}^{2}$ by an $\dot{H}^{\frac{1}{4}}$ norm. Let us consider the $\mathbb{R}^{n}$ case: assume we apply our estimate to a spectrally localized data $\Delta_{j} u_{0}$ : then, on the right-hand side,

$$
\left\|\Delta_{j} u_{0}\right\|_{L_{x}^{2}}^{2}\left\|\Delta_{j} u_{0}\right\|_{\dot{H}^{\frac{1}{2}}}^{2} \sim 2^{j}\left\|\Delta_{j} u_{0}\right\|_{L_{x}^{2}}^{4} .
$$

On the left-hand side, we get, summing in $j$,

$$
\sum_{j}\left\|\left|\nabla_{x}\right|^{\frac{3-n}{2}}\left(\left|\Delta_{j} u\right|^{2}\right)\right\|_{L_{t, x}^{2}} \lesssim\left\|u_{0}\right\|_{\dot{H}^{\frac{1}{4}}}^{2}
$$


As

$$
\left\|\sum_{j}\left|\Delta_{j} u\right|^{2}\right\|_{\dot{H} \frac{3-n}{2}} \lesssim \sum_{j}\left\|\left|\nabla_{x}\right|^{\frac{3-n}{2}}\left(\left|\Delta_{j} u_{0}\right|^{2}\right)\right\|_{L_{t, x}^{2}},
$$

when $n=3$ we are done, using the equivalence of the $L^{p}$ norm of $u$ with the $L^{p}$ norm of its square function, for $p=4$. For $n \neq 3$, one may decompose $|u|^{2}$ as a sum of a paraproduct and a reminder: our previous computation deals with the reminder, while the paraproduct term can be dealt with by applying the bilinear version of the estimate to $\Delta_{j} u$ and $\Delta_{k} u$ with $k \ll j$. We leave the details to the reader. The case of the exterior domain is dealt with in a similar way (using the Dirichlet Laplacian spectral localization!).

Remark 5.2. - Here and hereafter we define the fractional Sobolev spaces through the spectral localization. They do coincide with the usual ones in the range we are interested in, see [28], and the usual properties of the square function extend as well. Alternatively, one could define the localization through the heat flow and re-derive all required properties by hand, or define all spaces by summing a part which is localized close to the boundary (for which one may use all the known spectral properties on a bounded domain) and a part which is localized away from the boundary (and therefore belongs to the usual spaces defined on $\mathbb{R}^{n}$ ). This latter approach is essentially a poor man's version of the (spatial) localization property of Triebel-Lizorkin spaces, a key point in [28].

Now we proceed with the bounded domain $\Omega$ : call $v$ the extension of $u$ by 0 outside $\Omega$. Then we just proved

$$
\left\||\nabla|^{\frac{3-n}{2}}\left(|v|^{2}\right)\right\|_{L^{2}\left(0, T ; L^{2}\right)}^{2} \lesssim \sup _{(0, T)}\left(\|u\|_{2}^{2}\left(\|u\|_{2}\|u\|_{\dot{H}^{1}}+T\|u\|_{H^{1}}^{2}\right)\right) .
$$

Now, assume $u$ to be spectrally localized at (dyadic) $N: u=\phi\left(N^{-2} \Delta\right) u$ with $\phi \in C_{0}^{\infty}$ and the operator $\phi(\Delta)$ is defined by functional calculus through the spectral measure. Now, picking $T$ to be of size $N^{-1}$, the right-hand side in (5.6) will be bounded by $\|u\|_{H^{\frac{1}{4}}}^{4}$. Consider an interval $[0,1]$; by subdivision, one gets

$$
\left\||\nabla|^{\frac{3-n}{2}}\left(|v|^{2}\right)\right\|_{L^{2}\left(0,1 ; L^{2}\right)}^{2} \lesssim N \sup _{(0,1)}\|u\|_{H^{\frac{1}{4}}}^{4} \lesssim\|u\|_{H^{\frac{1}{2}}}^{4} .
$$

Now, by [28], the Sobolev norm of $|u|^{2}$ is equally the infimum over all extensions to $\mathbb{R}^{n}$, hence

$$
\left\||\nabla|^{\frac{3-n}{2}}\left(|u|^{2}\right)\right\|_{L^{2}\left(0,1 ; L^{2}(\Omega)\right)}^{2} \lesssim N \sup _{(0,1)}\|u\|_{H^{\frac{1}{4}}}^{4} \lesssim\left\|u_{0}\right\|_{H^{\frac{1}{2}}}^{4} \cdot
$$

Finally, one may freely pass from this inequality (which holds for a spectrally localized function $u$ ) to the general one with $u_{0} \in H^{\frac{1}{2}}(\Omega)$ by summing the dyadic pieces (built on the spectral localization).

\subsection{Scattering in $\mathbb{R}^{n}$}

Rather than developing the entire theory for all nonlinearities with $1+\frac{4}{n}<p<1+$ $\frac{4}{n-2}$, we focus on a couple of explicit examples. We feel that they are generic and provide a straightforward illustration of required techniques. As on a domain, we set

$$
M=\int|u|^{2} d x \text { and } E=\frac{1}{2} \int|\nabla u|^{2} d x+\frac{1}{p+1} \int|u|^{p+1} d x
$$


which are both conserved quantities. Consider, for $n=2$, and on $\mathbb{R}^{2}$,

$$
i \partial_{t} u+\Delta u=|u|^{4} u, \text { with } u_{\mid t=0}=u_{0} \in H^{1} .
$$

Local well-posedness can easily be obtained for $\dot{H}^{\frac{1}{2}}$ datum, and scattering requires control of appropriate space-time norms. An important feature of the local well-posedness result is that one may use Sobolev embedding in the course of the proof (as a consequence of the supercritical exponent with respect to $L^{2}$ ). On the other hand, our a priori bound (2.9), together with Sobolev embedding, yields

$$
\|u\|_{L_{t}^{4} L_{x}^{8}} \lesssim E^{\frac{1}{8}} M^{\frac{3}{8}}
$$

By Gagliardo-Nirenberg inequality, using $u \in L_{t}^{\infty}\left(\dot{H}^{1}\right)$, one gets

$$
\|u\|_{L_{t}^{6} L_{x}^{12}} \lesssim E^{\frac{1}{4}} M^{\frac{1}{4}}
$$

and this quantity scales like the $L_{t}^{\infty}\left(\dot{H}^{\frac{1}{2}}\right)$ norm, which is the scaling invariant norm. Hence, by Hölder and the Leibniz rule, using the $L_{t}^{6} L_{x}^{12}$ norm on four factors and either $L_{t}^{\infty}\left(L_{x}^{2}\right)$ or $L_{t}^{\infty}\left(\dot{H}^{1}\right)$ norm on one factor, we get two bounds

$$
\left\||u|^{4} u\right\|_{L_{t}^{\frac{3}{2}}\left(L_{x}^{\frac{6}{5}}\right)} \lesssim E M^{\frac{3}{2}} \text { and }\left\||u|^{4} u\right\|_{L_{t}^{\frac{3}{2}}\left(\dot{W}_{\frac{6}{5}}^{1}\right)} \lesssim E^{\frac{3}{2}} M
$$

Scattering in $L^{2}$ and $H^{1}$ follows immediately by Duhamel, as $\left(\frac{3}{2}, \frac{6}{5}\right)$ is a sharp Strichartz admissible pair. By interpolation, one can obtain scattering for all $H^{s}$ with $0<s<1$.

One may want to take advantage of the nonlinear part of our a priori bound (2.9); in fact, one has

$$
\left\||u|^{4}\right\|_{L_{t}^{2}\left(\dot{H}^{-\frac{1}{2}}\right)} \lesssim E^{\frac{1}{4}} M^{\frac{3}{4}}
$$

Combining this with the energy bound, one may prove that

$$
\left\||u|^{4} u\right\|_{L_{t}^{2} \dot{B}_{1}^{\frac{1}{2}, 2}} \lesssim E^{\frac{5}{4}} M^{\frac{3}{4}} .
$$

If the end-point Strichartz estimate were true with $n=2$, then one gets a better polynomial bound than the previous one. The lack of the end point may be routed around to obtain the last bound, with a small $\epsilon$ loss in the power of $M$.

We now provide another example, which illustrates that one may not always use the nonlinear part, and that estimating "in one shot" the right space-time norm is not necessarily doable, especially when close to the $L^{2}$-critical case. Consider, for $n=1$, and on $\mathbb{R}$,

$$
i \partial_{t} u+\Delta u=|u|^{5} u, \text { with } u_{\mid t=0}=u_{0} \in H^{1} .
$$

Local well-posedness can easily be obtained for $\dot{H}^{\frac{3}{10}}$ datum. The linear part of our a priori bound (2.9) can be interpolated with the mass conservation, and yields

$$
\left\||u|^{2}\right\|_{L_{t}^{3} L_{x}^{\infty}} \lesssim E^{\frac{1}{6}} M^{\frac{5}{6}}
$$

Hence, by product laws, using the $L_{t}^{6} L_{x}^{\infty}$ norm on three factors, the mass on one and Duhamel, we get (estimating the nonlinear part in $L_{t}^{1} L_{x}^{2}$, which may not be the optimal choice!)

$$
\|u\|_{L^{4}\left(t_{1}, t_{2} ; L_{x}^{\infty}\right)} \lesssim M^{\frac{1}{2}}+\|u\|_{L^{6}\left(t_{1}, t_{2} ; L_{x}^{\infty}\right)}^{3}\|u\|_{L^{4}\left(t_{1}, t_{2} ; L_{x}^{\infty}\right)}^{2} M^{\frac{1}{2}} .
$$

Assume that $\left(t_{1}, t_{2}\right)$ is such that

$$
\|u\|_{L^{6}\left(t_{1}, t_{2} ; L_{x}^{\infty}\right)}^{3} M^{\frac{1}{2}} \lesssim 1 / 10
$$


then

$$
\|u\|_{L^{4}\left(t_{1}, t_{2} ; L_{x}^{\infty}\right)} \lesssim 2 M^{\frac{1}{2}}
$$

Splitting the $L_{t}^{6}\left(L_{x}^{\infty}\right)$ in a finite number $N$ of small increments of equal size $S$, such that

$$
S^{6} M \sim 10^{-2} \text { and } N S^{6}=\|u\|_{L_{t}^{6}\left(L_{x}^{\infty}\right)}^{6},
$$

one controls the $L_{t}^{4} L_{x}^{\infty}$ norm, and the number of increments is $N=100 M\|u\|_{L_{t}^{6} L_{x}^{\infty}}^{6}$; namely,

$$
\|u\|_{L_{t}^{4}\left(L_{x}^{\infty}\right)}^{4} \lesssim 16 N M^{2} \lesssim M^{3}\left(E^{\frac{1}{6}} M^{\frac{5}{6}}\right)^{3}
$$

and finally

$$
\|u\|_{L_{t}^{4}\left(L_{x}^{\infty}\right)} \lesssim M^{\frac{11}{8}} E^{\frac{1}{8}}
$$

Scattering in $L_{x}^{2}$ follows immediately by Duhamel. Now, scattering in $\dot{H}^{1}$ follows by the exact same computation, using Leibniz rule, namely

$$
\left\|\partial_{x} u\right\|_{L^{4}\left(t_{1}, t_{2} ; L_{x}^{\infty}\right)} \lesssim E^{\frac{1}{2}}+\|u\|_{L^{6}\left(t_{1}, t_{2} ; L_{x}^{\infty}\right)}^{3}\|u\|_{L^{4}\left(t_{1}, t_{2} ; L_{x}^{\infty}\right)}^{2} E^{\frac{1}{2}}
$$

by interpolation, one may then obtain scattering in any $\dot{H}^{s}$ for $0<s<1$. On the other hand, attempts to use the $\int_{t, x}|u|^{9}$ nonlinear a priori bound seem to be doomed by scaling considerations. For $p \geq 13$, however, it becomes immediately relevant (notice that for $p=13$, $(p+3) /(p-1)=4 / 3)$.

REMARK 5.3. - Informally, for all dimensions $n \geq 1$ one may obtain scattering of the full range $1+\frac{4}{n}<p<1+\frac{4}{n-2}$ from (the linear part of) estimate (2.9). This can be seen through scaling considerations: one is given an a priori space-time bound at the level of the $\dot{H}^{\frac{1}{4}}$ norm. Through interpolation with the relevant bound (either energy or mass), one retrieves a scaleinvariant space-time bound. As the equation is $L^{2}$-supercritical, the fixed point argument is using even a tiny bit of Sobolev embedding to estimate the nonlinearity, and this is enough to insert the a priori estimate and close a true scale invariant Strichartz bound.

\subsection{Nonlinear equation on a domain}

We first deal with Theorem 3.4. Notice that the interesting case is $3 \leq p<5$, and we assume for the rest of the proof that $p$ is close to 5 , which is the most difficult case. Let us set notations: for any $1 \leq q \leq+\infty, L_{t}^{q}$ denotes a global in time norm, while $L_{T}^{q}$ denotes the norm on a finite time interval $(0, T)$. Moreover, any implicit constant in a $\lesssim$ sign does not depend on $T$ (in other words, time dependence is explicitly tracked).

We start with linear estimates on the homogeneous and inhomogeneous equation.

Lemma 5.4. - Let $S(t)$ denote the linear flow for the Schrödinger equation on an exterior domain $\Omega$ which satisfies the non trapping condition and let $s \geq 0$. Then,

$$
\left\|S(t) u_{0}\right\|_{L_{t}^{4}\left(\dot{W}_{0}^{s, 4}\right)} \lesssim\left\|u_{0}\right\|_{\dot{H}_{0}^{s+\frac{1}{4}}(\Omega)} .
$$

Denote by $w$ the solution of the inhomogeneous equation, e.g. $w=\int_{0}^{t} S(t-s) f(s) d s$,

$$
\|w\|_{C_{t}\left(\dot{H}_{0}^{s+\frac{1}{2}}\right)}+\|w\|_{L_{t}^{4}\left(\dot{W}^{s, 4}\right)} \lesssim\|f\|_{L_{t}^{\frac{4}{3} \dot{W}_{\frac{4}{3}}^{s+\frac{1}{2}}}} .
$$


Let $\chi_{1}$ and $\chi_{2}$ be two smooth cut-off functions which are such that $\chi_{1}=1$ on a ball $B_{1}$ such that $\mathbb{R}^{3} \backslash \Omega \subset B_{1}, \chi_{1}=0$ outside of $2 B_{1}$ and $\chi_{2}=1$ on $8 B_{1}, \chi_{2}=0$ outside $9 B_{1}$. Then

$$
\left\|\chi_{2} S(t) u_{0}\right\|_{L_{t}^{2}\left(H^{\frac{3}{2}}\right)}+\left\|\left(1-\chi_{1}\right) S(t) u_{0}\right\|_{L_{t}^{4}\left(L_{x}^{\infty}\right)} \lesssim\left\|u_{0}\right\|_{H_{0}^{1}}
$$

and

$$
\|w\|_{L_{t}^{4}\left(\dot{W}_{0}^{\frac{3}{4}, 4}\right)}+\left\|\chi_{2} w\right\|_{L_{t}^{2}\left(H^{\frac{3}{2}}\right)}+\left\|\left(1-\chi_{1}\right) w\right\|_{L_{t}^{4}\left(L_{x}^{\infty}\right)} \lesssim\|f\|_{L_{t}^{1}\left(H_{0}^{1}\right)} .
$$

Recall that from Theorem 2.6, we have an estimate on the linear flow $S(t)$ :

$$
\left\|S(t) u_{0}\right\|_{L_{t, x}^{4}} \lesssim\left\|u_{0}\right\|_{\dot{H}^{\frac{1}{4}}(\Omega)} .
$$

One may shift regularity by $s$ and obtain (5.9) using fractional powers of the Laplacian and equivalence of norms on domains ([28]); by the standard $T T^{\star}$ argument, we also obtain (5.10), which may again be shifted in regularity should it be necessary.

REMARK 5.5. - Notice that (5.9) barely fails to provide control of $L_{t}^{4}\left(L_{x}^{\infty}\right)$. One has to find an appropriate way to turn around this problem in order to deal with the nonlinear equation. Informally, one may use local smoothing estimates close to the boundary, and Strichartz estimate for the usual Laplacian on $\mathbb{R}^{3}$ away from it. The subcriticality with respect to $H^{1}$ of the nonlinear equation will compensate the weakness of the local smoothing estimate.

Let us now prove (5.11). Notice that multiplying by $\chi_{1}$ or $\chi_{2}$ localizes close to $\partial \Omega$ and $\chi_{2}=1$ on the support of $\chi_{1}$. Denote $u_{L}=S(t) u_{0}$. Then, the estimate on $\chi_{2} u_{L}$ in (5.11) follows immediately, by local smoothing (see [5]).

Consider now $\left(1-\chi_{1}\right) u_{L}$ : it solves

$$
i \partial_{t}\left(1-\chi_{1}\right) u_{L}+\Delta\left(1-\chi_{1}\right) u_{L}=\left[\chi_{1}, \Delta\right] u_{L},
$$

and the equation on the left is now set on $\mathbb{R}^{3}$. We proceed with a useful abstract lemma which is a simple consequence of a maximal function estimate due to Christ and Kiselev ([8]. See also [7] for a direct proof without Whitney decompositions).

Lemma 5.6. - Let $U(t)$ be a one parameter group of operators, $1 \leq r<q \leq+\infty$, $H$ an Hilbert space and $B_{r}$ and $B_{q}$ two Banach spaces. Suppose that

$$
\|U(t) \varphi\|_{L_{t}^{q}\left(B_{q}\right)} \lesssim\|\varphi\|_{H} \text { and }\left\|\int_{s} U(-s) g d s\right\|_{H} \lesssim\|g\|_{L_{t}^{r}\left(B_{r}\right)}
$$

then

$$
\left\|\int_{s<t} U(t-s) g(s) d s\right\|_{L_{t}^{q}\left(B_{q}\right)} \lesssim\|g\|_{L^{r}\left(B_{r}\right)} .
$$

Now, we pick $U(t)=S(t), L_{t}^{q}\left(B_{q}\right)=L_{t}^{3}\left(\dot{W}^{1, \frac{18}{5}}\right), L_{t}^{r}\left(B_{r}\right)=L_{t}^{2}\left(H_{\text {comp }}^{\frac{1}{2}}\right)$ and $H=H_{0}^{1}$. Then the homogeneous estimate in Lemma 5.6 is a Strichartz estimate (in $\mathbb{R}^{3}$, with Strichartz pair $\left(3, \frac{18}{5}\right)$ ) while the inhomogeneous estimate is the dual version of the local smoothing (shifted at the right regularity, see again [5]). Therefore, applying it to the inhomogeneous part of the solution to (5.14), we get

$$
\left\|\left(1-\chi_{1}\right) u_{L}\right\|_{L_{t}^{3}\left(\dot{W}^{1, \frac{18}{5}}\right)} \lesssim\left\|u_{0}\right\|_{H_{0}^{1}}+\left\|\left[\chi_{1}, \Delta\right] u_{L}\right\|_{L_{t}^{2}\left(\dot{H}^{\frac{1}{2}}\right)} .
$$


For a given function $\phi$, Gagliardo-Nirenberg inequality reads

$$
\|\phi\|_{L_{x}^{\infty}} \lesssim\|\phi\|_{L_{x}^{6}}^{\frac{1}{4}}\|\phi\|_{\dot{W}^{1, \frac{18}{5}}}^{\frac{3}{4}},
$$

which yields

$$
\left\|\left(1-\chi_{1}\right) u_{L}\right\|_{L_{t}^{4} L_{x}^{\infty}} \lesssim\left\|\left(1-\chi_{1}\right) u_{L}\right\|_{L_{t}^{\infty} \dot{H}_{0}^{1}}^{\frac{1}{4}}\left\|\left(1-\chi_{1}\right) u_{L}\right\|_{L_{t}^{3} \dot{W}^{1, \frac{18}{5}}}^{\frac{3}{4}},
$$

and

$$
\left\|\left(1-\chi_{1}\right) u_{L}\right\|_{L_{t}^{4}\left(L_{x}^{\infty}\right)} \lesssim\left\|u_{0}\right\|_{H_{0}^{1}}+\left\|\left[\chi_{1}, \Delta\right] u_{L}\right\|_{L_{t}^{2}\left(\dot{H}^{\frac{1}{2}}\right)} .
$$

Finally, as $\left\|\left[\chi_{1}, \Delta\right] u_{L}\right\|_{L_{t}^{2}\left(\dot{H}^{\frac{1}{2}}\right)} \lesssim\left\|\chi_{2} u_{L}\right\|_{L_{t}^{2}\left(\dot{H}^{\frac{3}{2}}\right)}$, we have obtained

$$
\left\|\left(1-\chi_{1}\right) u_{L}\right\|_{L_{t}^{4}\left(L_{x}^{\infty}\right)} \lesssim\left\|u_{0}\right\|_{H_{0}^{1}}
$$

Consider the inhomogeneous equation, $i \partial w+\Delta w=f$, with $w_{\mid t=0}=0$. Assume $f \in L_{T}^{1}\left(H_{0}^{1}\right)$, then by using local smoothing and our Strichartz estimate (5.9) on $S(t)$ (with $\left.s=\frac{3}{4}\right)$, and the Duhamel representation of $w$, we get

$$
\left\|\chi_{2} w\right\|_{L_{T}^{2}\left(H^{\frac{3}{2}}\right)}+\|w\|_{L_{T}^{4}\left(W^{\frac{3}{4}, 4}\right)} \lesssim\|f\|_{L_{T}^{1}\left(H_{0}^{1}\right)} .
$$

Again, consider $\left(1-\chi_{1}\right) w$, solution to

$$
i \partial_{t}\left(1-\chi_{1}\right) w+\Delta\left(1-\chi_{1}\right) w=\left[\chi_{1}, \Delta\right] w+\left(1-\chi_{1}\right) f
$$

exactly as before we get

$$
\left\|\left(1-\chi_{1}\right) w\right\|_{L_{T}^{4}\left(L_{x}^{\infty}\right)} \lesssim\|f\|_{L_{T}^{1}\left(H_{0}^{1}\right)}
$$

which ends the proof of Lemma 5.4.

We are now ready to set up a fixed point procedure for Equation (2.15) in the Banach space

$$
X=\left\{u \text { s.t. } u \in C_{T}\left(H_{0}^{1}\right) \cap L_{T}^{4}\left(W^{\frac{3}{4}, 4}\right), \quad \chi_{2} u \in L_{T}^{2}\left(H^{\frac{3}{2}}\right), \quad\left(1-\chi_{1}\right) u \in L_{T}^{4}\left(L_{x}^{\infty}\right)\right\} .
$$

Such a fixed point is standard (and will be omitted) once the following lemma is proven.

Lemma 5.7. - Let $f=|u|^{p-1} u-|v|^{p-1} v$ with $u, v \in X$. Then, for $p<5$,

$$
\|f\|_{L_{T}^{1}\left(H_{0}^{1}\right)} \lesssim T^{0^{+}}\|u-v\|_{X}\left(\|u\|_{X}^{p-1}+\|v\|_{X}^{p-1}\right) .
$$

Introduce $\chi_{3}=1$ on $4 B_{1}, \chi_{3}=0$ outside $5 B_{1}$. Let us start with $\chi_{3} f$ : due to the support conditions, one may replace $u$ and $v$ by $\chi_{2} u$ and $\chi_{2} v$ for as many factors as we wish. By interpolation between $L_{T}^{\infty}\left(H^{1}\right)$ and $L_{T}^{2}\left(H^{\frac{3}{2}}\right)$ for $\chi_{2} u$ and interpolation between $L_{T}^{\infty}\left(H^{1}\right)$ and $L_{T}^{4}\left(W^{\frac{3}{4}, 4}\right)$ for $u$, we have

$$
\left\|\chi_{2} u\right\|_{L_{T}^{m}\left(H^{\left.1+\frac{1}{m}\right)}\right.}+\|u\|_{L_{T}^{q}\left(L^{3 r}\right)} \lesssim\|u\|_{X},
$$

where $\frac{1}{r}=\frac{1}{2}-\frac{2}{q}$, and $m$ (resp. $q$ ) is to be thought of as very large (resp. slightly larger than 4). We proceed using $H^{1+\frac{1}{m}} \hookrightarrow W^{1, \lambda}$ with $1 / \lambda=1 / 2-1 /(3 m)$ and evaluate $\nabla\left(\chi_{3} f\right)$ : by chain rule, we are left with $\nabla\left(\chi_{2} g\right)\left(\chi_{2} h\right)^{p-1}$ where $g, h$ may be $u, v$ or $u-v$ (with one factor of $u-v$ in the $p$ factors). By Hölder, we obtain

$$
\left\|\nabla \chi_{3} f\right\|_{L_{T}^{\rho}\left(L_{x}^{2}\right)} \lesssim\left\|\chi_{2} g\right\|_{L_{T}^{m}\left(W^{1, \lambda}\right)}\left\|\chi_{2} h\right\|_{L_{T}^{q}\left(L_{x}^{3 r}\right)}^{p-1}
$$


with

$$
\frac{1}{\rho}=\frac{1}{m}+\frac{p-1}{q} \text { and } \frac{1}{m}=\frac{p-1}{r} .
$$

Let $p-1=4-\varepsilon$, and pick $m$ such that $\varepsilon>2 / m$, then $\rho>1$ and we recover the correct mapping, with a factor $T^{1-\frac{1}{\rho}}$ and $1-\frac{1}{\rho}=\frac{\varepsilon}{4}-\frac{1}{2 m}$ :

$$
\left\|\chi_{3} f\right\|_{L_{T}^{1}\left(H_{0}^{1}\right)} \lesssim T^{1-\frac{1}{\rho}}\left\|\chi_{2}(u-v)\right\|_{X}\left(\left\|\chi_{2} u\right\|_{X}^{p-1}+\left\|\chi_{2} v\right\|_{X}^{p-1}\right) .
$$

We now turn to $\left(1-\chi_{3}\right) f$; exactly as before, one may consider $\left(1-\chi_{1}\right) u$ and $\left(1-\chi_{1}\right) v$ rather than $u$ and $v$. Then one has trivially

$$
\left\|\left(1-\chi_{3}\right) f\right\|_{L_{T}^{1}\left(H_{0}^{1}\right)} \lesssim T^{\frac{\varepsilon}{4}}\left\|\left(1-\chi_{1}\right)(u-v)\right\|_{X}\left(\left\|\left(1-\chi_{1}\right) u\right\|_{X}^{p-1}+\left\|\left(1-\chi_{1}\right) v\right\|_{X}^{p-1}\right),
$$

using $u, v \in L_{T}^{\infty}\left(H_{0}^{1}\right)$ on one factor and $\left(1-\chi_{1}\right)(u, v) \in L_{T}^{4}\left(L_{x}^{\infty}\right)$ on the $4-\varepsilon$ remaining factors. This achieves the proof of Lemma 5.7.

Local existence and uniqueness in $X$ follows by standard arguments. Moreover, the local time of existence $T$ is such that

$$
T^{\frac{\varepsilon}{4}-\frac{1}{2 m}}\left\|u_{0}\right\|_{H_{0}^{1}}^{4-\varepsilon} \lesssim 1
$$

and one may use the conservation of energy to obtain global existence in the defocusing case. This achieves the proof of Theorem 3.4.

We turn to the scattering problem. As in the previous section, we only provide an explicit example rather than the best possible general case, for the sake of the exposition. We consider the defocusing cubic equation on a $3 \mathrm{D}$ exterior of a domain $\Omega$,

$$
i \partial_{t} u+\Delta u=|u|^{2} u, \text { with } u_{\mid \partial \Omega}=0, u_{t=0}=u_{0} \in H_{0}^{1}(\Omega),
$$

and require $\Omega$ to be star-shaped. Let $\chi_{1}, \chi_{2}, \chi_{3}$ be smooth cut-off functions close to the boundary $\partial \Omega$, with $\chi_{1} \leq \chi_{3} \leq \chi_{2}$ as in the existence proof we just completed.

Recall that we have two different nonlinear estimates which are valid for all times.

- From Proposition 2.7, we control a local smoothing type quantity at the level of $H^{\frac{1}{2}}$ regularity on the data:

$$
\int_{0}^{+\infty}\left\|\chi_{2} u\right\|_{\dot{H}_{0}^{1}}^{2} d t \lesssim M\left(u_{0}\right)^{\frac{1}{2}} E^{\frac{1}{2}}\left(u_{0}\right) .
$$

- From Proposition 2.9, we control a Strichartz-like norm, at the level of regularity $H^{\frac{1}{4}}$ on the data:

$$
\|u\|_{L_{t}^{4}\left(L^{4}(\Omega)\right)} \lesssim M^{\frac{3}{8}} E^{\frac{1}{8}} .
$$

Ultimately, we aim at controlling space-time norms at the level of $H^{1}$ regularity on the data. We start by bootstrapping our relatively weak control (5.27) into a somewhat stronger estimate at the level of $H^{\frac{1}{2}}$ regularity.

Lemma 5.8. - Let u be a solution of (5.25). Then

$$
\chi_{1} u \in L_{t}^{4}\left(\dot{W}^{\frac{1}{4}, 4}\right) \text { and }\left(1-\chi_{1}\right) u \in L_{t}^{3}\left(\dot{W}^{\frac{1}{2}, \frac{18}{5}}\right) \cap L_{t}^{\frac{12}{5}}\left(\dot{W}^{\frac{1}{2}, \frac{9}{2}}\right) .
$$

As a consequence, the solution $u$ scatters in $H^{\frac{1}{2}}$. 
In order to prove the lemma, we again split the equation, treating differently the neighborhood of the boundary (where local smoothing is most efficient) and spatial infinity (where Strichartz estimates for the free propagator are available). Consider $\chi_{1} u$, which is a solution to

$$
i \partial_{t} \chi_{1} u+\Delta \chi_{1} u=\chi_{1}|u|^{2} u-\left[\chi_{1}, \Delta\right] u=f .
$$

On the nonlinear part, which is compactly supported, we use (5.26) on one factor, and $L_{t}^{\infty}\left(H_{0}^{1}\right)$ on the other two, while the commutator term is easily controlled by $\left\|\chi_{2} u\right\|_{L^{2}\left(\dot{H}_{0}^{1}\right)}$; hence, globally in time,

$$
\|f\|_{L_{t}^{2}\left(L_{\text {comp }}^{2}\right)} \lesssim\left\|\chi_{2} u\right\|_{L_{t}^{2}\left(\dot{H}_{0}^{1}\right)}\left(E\left(u_{0}\right)+1\right) .
$$

Then, we apply Lemma 5.6, this time with $H=\dot{H}^{\frac{1}{2}}, L_{t}^{q}\left(B_{q}\right)=L_{t}^{4}\left(\dot{W}^{\frac{1}{4}, 4}\right)$ (this from interpolation between (5.9) and (5.13)) and $L_{t}^{p}\left(B_{p}\right)=L_{t}^{2}\left(L_{\text {comp }}^{2}\right)$ (which is dual local smoothing at regularity $\left.H^{\frac{1}{2}}\right)$; we obtain $\chi_{1} u \in L_{t}^{4}\left(\dot{W}^{\frac{1}{4}, 4}\right)$.

Let us deal with $\left(1-\chi_{1}\right) u$, which is solution to

$$
i \partial_{t}\left(1-\chi_{1}\right) u+\Delta\left(1-\chi_{1}\right) u=\left(1-\chi_{1}\right)|u|^{2} u+\left[\chi_{1}, \Delta\right] u .
$$

Recall that we are now on the whole space $\mathbb{R}^{3}$. The commutator term is dealt with exactly as with the previous part, and is therefore $L_{t}^{2}\left(L_{\text {comp }}^{2}\right)$. Another application of Lemma 5.6 but with $L_{t}^{q}\left(B_{q}\right)$ being either $L_{t}^{3}\left(\dot{W}^{\frac{1}{2}, \frac{18}{5}}\right)$ or $L_{t}^{\frac{12}{5}}\left(\dot{W}^{\frac{1}{2}, \frac{9}{2}}\right)$ (both $\left(3, \frac{18}{5}\right)$ and $\left(\frac{12}{5}, \frac{9}{2}\right)$ are Strichartz pairs for the free space) yields the claim for the Duhamel term coming from the commutator. One the other hand,

$$
\left\|\left(1-\chi_{1}\right)|u|^{2} u\right\|_{L_{t}^{2}\left(\dot{W}^{1,1}\right)} \lesssim\|u\|_{L_{t}^{\infty}\left(\dot{H}^{1}\right)}\|u\|_{L_{t, x}^{4}}^{2} \lesssim M^{\frac{3}{4}} E^{\frac{3}{4}},
$$

where we used our other a priori control (5.27) and the energy conservation. From the embedding $L_{t}^{2}\left(\dot{W}^{1,1}\right) \hookrightarrow L_{t}^{2}\left(\dot{W}^{\frac{1}{2}}, \frac{6}{5}\right)$, we may apply the dual end-point Strichartz estimate on the nonlinear term and finally $\left(1-\chi_{1}\right) u \in L_{t}^{3}\left(\dot{W}^{\frac{1}{2}, \frac{18}{5}}\right) \cap L_{t}^{\frac{12}{5}}\left(\dot{W}^{\frac{1}{2}, \frac{9}{2}}\right)$ which achieves the proof of (5.28).

From $\left(1-\chi_{1}\right) u \in L_{t}^{3}\left(\dot{W}^{\frac{1}{2}}, \frac{18}{5}\right) \hookrightarrow L^{3}\left(L_{x}^{9}\right)$, we infer by Leibniz, Hölder $\left(\frac{1}{2}=\frac{2}{9}+\frac{5}{18}\right)$ and interpolation,

$$
\left(\left(1-\chi_{1}\right) u\right)^{3} \in L_{t}^{1}\left(\dot{H}^{\frac{1}{2}}\right) .
$$

Going back to the equation on $u$ and splitting the source term $|u|^{2} u$ as $g_{1}=\chi_{2}|u|^{2} u$ and $g_{2}=$ $\left(1-\chi_{2}\right)|u|^{2} u$, we have for the same reason as before (and globally in time!) $g_{1} \in L_{t}^{2}\left(L_{\text {comp }}^{2}\right)$, while from (5.31), $g_{2} \in L_{t}^{1}\left(\dot{H}^{\frac{1}{2}}\right)$. Then, with $S(t)$ being the Schrödinger group on our domain,

$S(-t) u=u_{0}+\int_{0}^{t} S(-s)\left(g_{1}+g_{2}\right) d s=u_{0}+\int_{0}^{+\infty} S(-s)\left(g_{1}+g_{2}\right) d s-\int_{t}^{+\infty} S(-s)\left(g_{1}+g_{2}\right) d s$,

from which scattering in $\dot{H}^{\frac{1}{2}}$ follows: both integral terms are well-defined in $\dot{H}^{\frac{1}{2}}$, and the second one vanishes when $t \rightarrow+\infty$. This proves Lemma 5.8.

We aim at bootstrapping this information up to $H^{1}$ scattering in two steps. First, we improve our new space-time controls to the level of $\dot{H}^{\frac{3}{4}}$ regularity: once again, the important point is to use only global in time bounds. 
Lemma 5.9. - Let u be a solution of (5.25). Then

$$
\chi_{2} u \in L_{t}^{2}\left(\dot{H}^{\frac{5}{4}}\right) \text { and } u \in L_{t}^{4}\left(\dot{W}^{\frac{1}{2}, 4}\right) .
$$

The proof of Lemma 5.9 is more delicate than the previous one, as a splitting time argument (like we did for scattering in $\mathbb{R}^{n}$ ) is required. From the local existence theory, we can easily get that the $L_{T}^{4}\left(\dot{W}^{\frac{1}{2}, 4}\right)$ is finite for $T<+\infty$. We will prove that $T=+\infty$, by using the equation and Duhamel:

- we start with $g_{1}=\chi_{3}|u|^{2} u$ : interpolating between $u \in L_{t}^{\infty}\left(\dot{H}^{1}\right)$ and $\chi_{2} u \in L_{t}^{2}\left(\dot{H}^{1}\right)$, we have $\chi_{2} u \in L_{t}^{4}\left(\dot{H}^{1}\right)$. On the other hand, from Lemma 5.8, $u \in L_{t}^{4}\left(\dot{W}^{\frac{1}{4}, 4}\right)$; by interpolation with $u \in L_{T}^{4}\left(\dot{W}^{\frac{1}{2}, 4}\right)$ and Sobolev, we get $u^{2} \in L_{T}^{2}\left(L_{x}^{4}\right)$ and

$$
\left\|\chi_{3}|u|^{2} u\right\|_{L_{T}^{\frac{4}{3}\left(\dot{W}^{1, \frac{4}{3}}\right)}} \lesssim\|u\|_{L_{t}^{\infty}\left(\dot{H}^{1}\right)}^{\frac{1}{2}}\left\|\chi_{2} u\right\|_{L_{t}^{2}\left(\dot{H}^{1}\right)}^{\frac{1}{2}}\|u\|_{L_{t}^{4}\left(\dot{W}^{\frac{1}{4}, 4}\right)}\|u\|_{L_{T}^{4}\left(\dot{W}^{\frac{1}{2}, 4}\right)} ;
$$

- let us deal with $g_{2}=\left(1-\chi_{3}\right)|u|^{2} u$ : interpolating between $u \in L_{t}^{\infty}\left(\dot{H}^{1}\right)$ and $\left(1-\chi_{1}\right) u \in$ $L_{t}^{3}\left(\dot{W}^{\frac{1}{2}}, \frac{18}{5}\right)$ (which we got from Lemma 5.8), we obtain $\left(1-\chi_{1}\right) u \in L_{t}^{6}\left(\dot{W}^{\frac{3}{4}}, \frac{18}{7}\right)$. Recall as well that Lemma 5.8 provides $\left(1-\chi_{1}\right) u \in L_{t}^{\frac{12}{5}}\left(\dot{W}^{\frac{1}{2}, \frac{9}{2}}\right) \hookrightarrow L_{t}^{\frac{12}{5}}\left(L_{x}^{18}\right)$; using this information on two factors and the interpolation bound on the third one, we get

$$
\left\|\left(1-\chi_{3}\right)|u|^{2} u\right\|_{L_{t}^{1}\left(\dot{H}^{\frac{3}{4}}\right)} \lesssim\|u\|_{L_{t}^{\infty}\left(\dot{H}^{1}\right)}^{\frac{1}{2}}\left\|\left(1-\chi_{1}\right) u\right\|_{L_{t}^{3}\left(\dot{W}^{\frac{1}{2}, \frac{18}{5}}\right)}^{\frac{1}{2}}\left\|\left(1-\chi_{1}\right) u\right\|_{L_{t}^{\frac{12}{5}}\left(\dot{W}^{\frac{1}{2}, \frac{9}{2}}\right)}^{2} .
$$

Using the equation, Duhamel and (5.10) at regularity $s=\frac{1}{2}$, we have

$$
\|u\|_{L_{T}^{4}\left(\dot{W}^{\frac{1}{2}, 4}\right)} \lesssim\left[\left\|u_{0}\right\|_{\dot{H}^{\frac{3}{4}}}+\left\|\left(1-\chi_{3}\right)|u|^{2} u\right\|_{L_{t}^{1}\left(\dot{H}^{\frac{3}{4}}\right)}\right]+M^{\frac{1}{2}} E\|u\|_{L_{t}^{4}\left(\dot{W}^{\frac{1}{4}, 4}\right)}\|u\|_{L_{T}^{4}\left(\dot{W}^{\frac{1}{2}, 4}\right)} ;
$$

the bracket term is finite by (5.34), and a splitting time argument performed on the $L_{t}^{4}\left(\dot{W}^{\frac{1}{4}, 4}\right)$ norm which is finite provides global in time control of $u \in L_{t}^{4}\left(\dot{W}^{\frac{1}{2}, 4}\right)$. Using Duhamel, again, on $g_{1}$ and $g_{2}$, we also obtain $\chi_{3} u \in L_{t}^{2}\left(\dot{H}^{\frac{5}{4}}\right)$, globally in time (note that for $g_{1}$ we have to resort again to Lemma 5.6, combining (5.10) and local smoothing). This achieves the proof of Lemma 5.9. We finally need one last step.

Lemma 5.10. - Let $u$ be a solution of (5.25). Then

$$
\chi_{3}|u|^{2} u \in L_{t}^{\frac{4}{3}}\left(\dot{W}^{\frac{5}{4}, \frac{4}{3}}\right) \text { and }\left(1-\chi_{3}\right)|u|^{2} u \in L_{t}^{1}\left(\dot{H}_{0}^{1}\right) \text {. }
$$

Again, we proceed differently close to or far from the boundary.

- On $g_{1}$, we use $\chi_{2} u \in L_{t}^{2}\left(\dot{H}^{\frac{5}{4}}\right)$ from Lemma 5.9 and $u \in L_{t, x}^{8}$ (which follows from $u \in L_{t}^{\infty}\left(\dot{H}^{1}\right)$ and $u \in L_{t}^{4}\left(\dot{W}^{\frac{1}{2}, 4}\right)$, again from Lemma 5.9), and obtain

$$
\left\|g_{1}\right\|_{L_{t}^{\frac{4}{3}\left(\dot{W}^{\frac{5}{4}}, \frac{4}{3}\right)}} \lesssim\left\|\chi_{2} u\right\|_{L_{t}^{2}\left(\dot{H}^{\frac{5}{4}}\right)}\|u\|_{L_{t, x}^{8}}^{2} .
$$

- For $g_{2}$, we need $\left(1-\chi_{3}\right) u \in L_{t}^{2}\left(L_{x}^{\infty}\right)$ which does not follow from the Strichartz estimates we already obtained on $\left(1-\chi_{1}\right) u$ (missing end-point, not to mention a log). We use (5.30), but with the cut $\chi_{3}$ instead of $\chi_{1}$ :

$$
i \partial_{t}\left(1-\chi_{3}\right) u+\Delta\left(1-\chi_{3}\right) u=\left(1-\chi_{3}\right)|u|^{2} u+\left[\chi_{3}, \Delta\right] u \text {. }
$$

For the nonlinear part,

$$
\left\|\left(1-\chi_{3}\right)|u|^{2} u\right\|_{L_{t}^{2}\left(L_{x}^{\frac{6}{5}}\right)} \lesssim\|u\|_{L^{\infty}\left(\dot{H}^{\frac{1}{2}}\right)}\|u\|_{L_{t, x}^{4}}^{2} .
$$


The commutator term is $L_{t}^{2}\left(L_{\text {comp }}^{2}\right)$ hence $L_{t}^{2}\left(L_{x}^{\frac{6}{5}}\right)$ and by Duhamel (with the free propagator!) we get by Strichartz $\left(1-\chi_{3}\right) u \in L_{t}^{2}\left(L_{x}^{6}\right)$. On the other hand, using again (5.36), $\chi_{3} u \in L_{t}^{2}\left(\dot{H}^{\frac{5}{4}}\right)$ for the commutator term, and (5.34) for the nonlinear part, we wish to obtain by (free) Strichartz and Duhamel, $\left(1-\chi_{3}\right) u \in L_{t}^{2}\left(\dot{W}^{\frac{3}{4}, 6}\right)$. This is indeed the case for the Duhamel term coming from the nonlinear term. However, one may no longer use Lemma 5.6 for the commutator term, and we need in a crucial way the $L_{t}^{2}$ norm. Fortunately enough, we may use

Lemma 5.11 (Staffilani-Tataru [25]). - Let $x \in \mathbb{R}^{n}, n \geq 3$ and let $f(x, t)$ be compactly supported in space, such that $f \in L_{t}^{2}\left(H^{-\frac{1}{2}}\right)$. Then the solution $w$ to $\left(i \partial_{t}+\Delta_{x}\right) w=$ $f$ with $w_{\mid t=0}=0$, is such that

$$
\|w\|_{L_{t}^{2}\left(L_{x}^{\left.\frac{2 n}{n-2}\right)}\right.} \lesssim\|f\|_{L_{t}^{2}\left(H^{-\frac{1}{2}}\right)} .
$$

In other words, provided the left handside is a local smoothing norm, one recover the endpoint estimate in addition to the ones provided by Lemma 5.6. A quick inspection of the proof in [25] allows one to shift spatial regularity as we need.

Going back to our equation on $\left(1-\chi_{3}\right) u$, we therefore obtain $\left(1-\chi_{3}\right) u \in L_{t}^{2}\left(\dot{W}^{\frac{3}{4}}, 6\right)$; Gagliardo-Nirenberg immediately provides $\left(1-\chi_{3}\right) u \in L_{t}^{2}\left(L_{x}^{\infty}\right)$. Combining this with $u \in L_{t}^{\infty}\left(\dot{H}^{1}\right)$, we finally get $\left(1-\chi_{2}\right)|u|^{2} u \in L_{t}^{1}\left(\dot{H}^{1}\right)$. This achieves the proof of Lemma 5.10.

From the informations on $g_{1}$ and $g_{2}$ provided by Lemma 5.10, we may go back to (5.32) and obtain scattering in $H^{1}$ like we did in $H^{\frac{1}{2}}$. This achieves the proof of Theorem 3.5.

REMARK 5.12. - If one picks $p>3$, scattering in a negative regularity Sobolev space may easily be obtained. Bootstrapping appears to be more difficult, the numerology working in the wrong direction when $p$ gets closer to 5 , as both a priori bounds (the smoothing and the $L_{t, x}^{4}$ are subcritical with respect to scaling).

\section{REFERENCES}

[1] R. Anton, Global existence for defocusing cubic NLS and Gross-Pitaevskii equations in three dimensional exterior domains, J. Math. Pures Appl. 89 (2008), 335-354.

[2] M. D. Blair, H. F. Smith, C. D. Sogge, On Strichartz estimates for Schrödinger operators in compact manifolds with boundary (electronic), Proc. Amer. Math. Soc. 136 (2008), 247-256.

[3] J. Bourgain, Refinements of Strichartz' inequality and applications to 2D-NLS with critical nonlinearity, Int. Math. Res. Not. 1998 (1998), 253-283.

[4] N. BurQ, Smoothing effect for Schrödinger boundary value problems, Duke Math. J. 123 (2004), 403-427.

[5] N. Burq, P. GÉrard, N. Tzvetkov, On nonlinear Schrödinger equations in exterior domains, Ann. Inst. H. Poincaré Anal. Non Linéaire 21 (2004), 295-318.

[6] N. Burq, P. Gérard, N. Tzvetkov, Strichartz inequalities and the nonlinear Schrödinger equation on compact manifolds, Amer. J. Math. 126 (2004), 569-605.

$4^{\mathrm{e}}$ SÉRIE - TOME $42-2009-\mathrm{N}^{\mathrm{o}} 2$ 
[7] N. Burq, F. Planchon, Smoothing and dispersive estimates for 1D Schrödinger equations with BV coefficients and applications, J. Funct. Anal. 236 (2006), 265-298.

[8] M. Christ, A. Kiselev, Maximal functions associated to filtrations, J. Funct. Anal. 179 (2001), 409-425.

[9] J. Colliander, M. Grillakis, N. Tzirakis, The interaction Morawetz estimate for $\mathbb{R}^{2}$. (Workshop "Nonlinear waves and dispersive equations"), Oberwolfach Report 44 (2007).

[10] J. Colliander, M. Grillakis, N. Tzirakis, Tensor products and correlation estimates with applications to nonlinear Schrödinger equations, to appear in Comm. Pure Appl. Math.

[11] J. Colliander, J. Holmer, M. Visan, X. Zhang, Global existence and scattering for rough solutions to generalized nonlinear Schrödinger equations on $\mathbb{R}$, Commun. Pure Appl. Anal. 7 (2008), 467-489.

[12] J. Colliander, M. Keel, G. Staffilani, H. Takaoka, T. Tao, Global existence and scattering for rough solutions of a nonlinear Schrödinger equation on $\mathbb{R}^{3}$, Comm. Pure Appl. Math. 57 (2004), 987-1014.

[13] J. Colliander, M. Keel, G. Staffilani, H. Takaoka, T. TaO, Global wellposedness and scattering for the energy-critical nonlinear Schrödinger equation in $\mathbb{R}^{3}$, Ann. of Math. 167 (2008), 767-865.

[14] P. Constantin, J.-C. Saut, Local smoothing properties of Schrödinger equations, Indiana Univ. Math. J. 38 (1989), 791-810.

[15] J. Ginibre, G. Velo, Scattering theory in the energy space for a class of nonlinear Schrödinger equations, J. Math. Pures Appl. 64 (1985), 363-401.

[16] J. Ginibre, G. Velo, Quadratic Morawetz inequalities and asymptotic completeness in the energy space for nonlinear Schrödinger and Hartree equations, to appear in Quart. Appl. Math.

[17] A. Hassell, T. TaO, J. Wunsch, A Strichartz inequality for the Schrödinger equation on nontrapping asymptotically conic manifolds, Comm. Partial Differential Equations 30 (2005), 157-205.

[18] O. Ivanovici, Precised smoothing effect in the exterior of balls, Asymptot. Anal. 53 (2007), 189-208.

[19] M. Keel, T. Tao, Endpoint Strichartz estimates, Amer. J. Math. 120 (1998), 955-980.

[20] C. E. Kenig, G. Ponce, L. Vega, Oscillatory integrals and regularity of dispersive equations, Indiana Univ. Math. J. 40 (1991), 33-69.

[21] J. E. Lin, W. A. Strauss, Decay and scattering of solutions of a nonlinear Schrödinger equation, J. Funct. Anal. 30 (1978), 245-263.

[22] T. Ozawa, Y. Tsutsumi, Space-time estimates for null gauge forms and nonlinear Schrödinger equations, Differential Integral Equations 11 (1998), 201-222.

[23] D. SALORT, Dispersion and Strichartz inequalities for the one-dimensional Schrödinger equation with variable coefficients, Int. Math. Res. Not. 2005 (2005), 687-700.

[24] P. SıöLIN, Regularity of solutions to the Schrödinger equation, Duke Math. J. 55 (1987), 699-715. 
[25] G. Staffilani, D. Tataru, Strichartz estimates for a Schrödinger operator with nonsmooth coefficients, Comm. Partial Differential Equations 27 (2002), 1337-1372.

[26] R. S. Strichartz, Restrictions of Fourier transforms to quadratic surfaces and decay of solutions of wave equations, Duke Math. J. 44 (1977), 705-714.

[27] T. TaO, A. Vargas, L. Vega, A bilinear approach to the restriction and Kakeya conjectures, J. Amer. Math. Soc. 11 (1998), 967-1000.

[28] H. Triebel, Theory of function spaces, Monographs in Mathematics 78, Birkhäuser, 1983.

[29] L. Vega, Schrödinger equations: pointwise convergence to the initial data, Proc. Amer. Math. Soc. 102 (1988), 874-878.

[30] L. Vega, N. Visciglia, On the local smoothing for the Schrödinger equation (electronic), Proc. Amer. Math. Soc. 135 (2007), 119-128.

\author{
Fabrice Planchon \\ Laboratoire Analyse, Géométrie \& Applications \\ UMR 7539 du CNRS \\ Institut Galilée, Université Paris 13 \\ 99 av. J.-B. Clément \\ F-93430 Villetaneuse, France \\ E-mail: fab@math.univ-paris13.fr \\ Luis Vega \\ Universidad del Pais Vasco \\ Euskal Herriko Unibertsitatea \\ Departamento de Matematicas Aptdo. 644, \\ 48080 Bilbao, Spain \\ E-mail: luis.vega@ehu.es
}

